Article

\title{
Analysis of Multi-Antenna GNSS Receiver Performance under Jamming Attacks
}

\author{
Niranjana Vagle *, Ali Broumandan and Gérard Lachapelle \\ PLAN Group, Department of Geomatics Engineering, Schulich School of Engineering, University of Calgary, \\ 2500 University Drive N. W., Calgary, AB T2N 1N4, Canada; abrouman@ucalgary.ca (A.B.); \\ gerard.lachapelle@ucalgary.ca (G.L.) \\ * Correspondence: vaglen@ucalgary.ca; Tel.: +1-403-210-9798
}

Academic Editor: Vittorio M. N. Passaro

Received: 11 October 2016; Accepted: 11 November 2016; Published: 17 November 2016

\begin{abstract}
Although antenna array-based Global Navigation Satellite System (GNSS) receivers can be used to mitigate both narrowband and wideband electronic interference sources, measurement distortions induced by array processing methods are not suitable for high precision applications. The measurement distortions have an adverse effect on the carrier phase ambiguity resolution, affecting the navigation solution. Depending on the array attitude information availability and calibration parameters, different spatial processing methods can be implemented although they distort carrier phase measurements in some cases. This paper provides a detailed investigation of the effect of different array processing techniques on array-based GNSS receiver measurements and navigation performance. The main novelty of the paper is to provide a thorough analysis of array-based GNSS receivers employing different beamforming techniques from tracking to navigation solution. Two beamforming techniques, namely Power Minimization (PM) and Minimum Power Distortionless Response (MPDR), are being investigated. In the tracking domain, the carrier Doppler, Phase Lock Indicator (PLI), and Carrier-to-Noise Ratio $\left(\mathrm{C} / \mathrm{N}_{0}\right)$ are analyzed. Pseudorange and carrier phase measurement distortions and carrier phase position performance are also evaluated. Performance analyses results from simulated GNSS signals and field tests are provided.
\end{abstract}

Keywords: GNSS; array processing; measurement distortions; carrier phase positioning

\section{Introduction}

Applications of antenna arrays for mitigating different types of Global Navigation Satellite System (GNSS) interference have been widely studied [1-11]. Unlike other wireless applications, the quality of GNSS measurements after interference mitigation is important for high precision applications. However, antenna array processing induces distortions in code and carrier phase measurements in the presence of electronic interference [12-23]. The distortions in GNSS measurements caused by antenna array processing have been studied previously; for instance, [12,20] demonstrated distortions in carrier phase measurements with actual data and showed the dependency of distortions on satellite direction. In [13], code phase biases on the order of one to two metres using Monte-Carlo simulations in the presence of interference and mutual coupling were observed using the Minimum Variance Distortionless Response (MVDR) technique. In [8], authors studied phase tracking loop performance for possible carrier phase distortions due to different array processing techniques such as minimum variance and MVDR; it was shown through simulations that the minimum variance or power minimization based beamformers suffer comparatively more phase distortion than a MVDR based technique. Global Positioning System (GPS) phase measurement distortions due to phase centre variations of individual antenna elements in an array are studied in [18,19]. It has been shown that mutual coupling between antennas also causes phase distortions of a few centimetres; 
these are not tolerable for precise applications as they render carrier phase ambiguity resolution more difficult to achieve. In addition, code phase measurement biases due to the phase delay and group delay of antenna elements were observed. Although code phase and carrier phase measurement distortions were reported, a detailed analysis of these and navigation solution quality for high precision applications was not completed. In this research, the performance of a multi-antenna GNSS receiver is evaluated for different array processing techniques in terms of carrier tracking, measurement, and position distortions. Both narrow band and wide band interference sources are considered. Two array processing techniques, namely PM beamformer, which does not take Angle-of-Arrival (AOA) information of satellites into consideration, and MPDR beamformer, which uses the AOA of satellites [24], are considered. The methodology for evaluating possible measurement distortions is described and followed by a discussion of the results using simulated and actual data. Performance evaluations are carried out using GPS L1 Coarse/Acquisition (C/A) signals.

\section{Methodology}

This section describes GNSS and interference signal simulations, the multi-antenna signal processing method, and the methodology to characterize the code and carrier phase measurement distortions used in the sequel.

\subsection{Signal and System Model}

A rectangular planar array planar is used herein. Consider the case of an $M \times N$ element uniform rectangular array. The elements are lying in the $x-y$ plane and are equally spaced by $d_{m}$ in the $x$-direction and $d_{n}$ in the $y$-direction as shown in Figure 1.

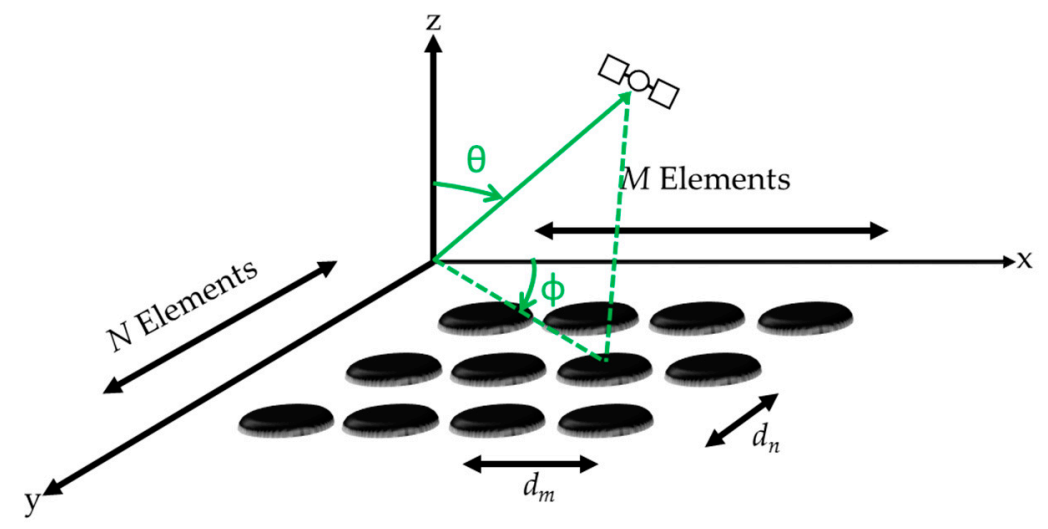

Figure 1. Rectangular array structure with $M \times N$ elements.

After down-conversion and sampling, the digitized signal received at the $(m, n)$ th antenna element can be expressed as

$$
\begin{aligned}
\mathrm{x}_{m, n}(t)= & \sum_{k=1}^{K} s_{k}(t) e^{j \frac{2 \pi}{\lambda}\left[(m-1) d_{m} \sin \left(\theta_{k}\right) \sin \left(\phi_{k}\right)+(n-1) d_{n} \sin \left(\theta_{k}\right) \cos \left(\phi_{k}\right)\right]} \\
& +\sum_{j=1}^{J} j_{j}(t) e^{j \frac{2 \pi}{\lambda}\left[(m-1) d_{m} \sin \left(\theta_{j}\right) \sin \left(\phi_{j}\right)+(n-1) d_{n} \sin \left(\theta_{j}\right) \cos \left(\phi_{j}\right)\right]}+\eta_{m, n}(t)
\end{aligned}
$$

where $s_{k}(t)$ is the $k$ th satellite signal component, $j_{j}(t)$ is the $j$ th electronic interference, $\lambda$ refers to the wavelength of the signal, $\left(\theta_{k}, \phi_{k}\right)$ is the elevation and azimuth angle of the $k$ th satellite, $\left(\theta_{j}, \phi_{j}\right)$ is the elevation and azimuth angle of the electronic interference signal, $K$ is the number of Pseudo Random Numbers (PRNs), $J$ is the number of electronic interference signals, and $\eta_{m, n}(t)$ is the additive spatially white noise of the $(m, n)$ th antenna element. 
The impinged signals from all the antenna elements can be represented in matrix form as

$$
\mathbf{x}=\mathbf{A s}+\mathbf{B} \mathbf{j}+\eta
$$

where $\mathbf{x}$ is the $M N \times 1$ received signal vector, $\eta$ is the $M N \times 1$ noise vector, $\mathbf{s}$ is the $K \times 1$ satellite signal vector, and $\mathbf{j}$ is the $J \times 1$ signal vector affected by electronic interference; these vectors are given by

$$
\begin{gathered}
\mathbf{x}=\left[\mathrm{x}_{1,1}(t), \mathrm{x}_{2,1}(t), \ldots, \mathrm{x}_{M, 1}(t), \mathrm{x}_{1,2}(t), \ldots, \mathrm{x}_{M, N}(t)\right]^{T} \\
\mathbf{s}=\left[s_{1}(t), s_{2}(t), \ldots, s_{K}(t)\right]^{T} \\
\mathbf{j}=\left[j_{1}(t), j_{2}(t), \ldots, j_{J}(t)\right]^{T} \\
\boldsymbol{\eta}=\left[\eta_{1,1}(t), \eta_{2,1}(t), \ldots \eta_{M, 1}(t), \eta_{1,2}(t), \ldots \eta_{M, N}(t)\right]^{T}
\end{gathered}
$$

The steering matrices $\mathbf{A}$ and $\mathbf{B}$ are of dimensions $M N \times K$ and $M N \times J$ respectively and are given by

$$
\begin{aligned}
& \mathbf{A}=\left[\mathbf{a}_{1}, \mathbf{a}_{2}, \ldots, \mathbf{a}_{k}\right]^{T} \\
& \mathbf{B}=\left[\mathbf{b}_{1}, \mathbf{b}_{2}, \ldots, \mathbf{b}_{j}\right]^{T}
\end{aligned}
$$

where $\mathbf{a}_{k}$ is the $M N \times 1$ steering vector of the $k$ th satellite signal component coming from direction $\left(\theta_{k}, \phi_{k}\right)$ and $\mathbf{b}_{j}$ is the $M N \times 1$ steering vector of the $j$ th electronic interference signal coming from direction $\left(\theta_{j}, \phi_{j}\right)$. Both $\mathbf{a}_{k}$ and $\mathbf{b}_{j}$ have a similar structure, hence only $\mathbf{a}_{k}$ is defined and is given by

$$
\begin{gathered}
\mathbf{a}_{k}=\left[\mathbf{c}_{k}^{T}, \gamma_{k} \mathbf{c}_{k}^{T}, \ldots, \gamma_{k}^{N-1} \mathbf{c}_{k}^{T}\right]^{T} \\
\mathbf{c}_{k}=\left[1, \beta_{k}, \ldots, \beta_{k}^{M-1}\right]^{T} \\
\gamma_{k}=e^{j \frac{2 \pi}{\lambda}\left[d_{n} \sin \left(\theta_{k}\right) \cos \left(\phi_{k}\right)\right]} \\
\beta_{k}=e^{j \frac{2 \pi}{\lambda}\left[d_{m} \sin \left(\theta_{k}\right) \sin \left(\phi_{k}\right)\right]}
\end{gathered}
$$

\subsection{Multi-Antenna Signal Simulations and Processing}

The multi-antenna GNSS signal simulator developed in [25] was used to generate multi-antenna signals with different types of interference. For the simulations, it was assumed that antenna elements are identical and there is no mutual coupling. A static user scenario was considered and the interference source was also assumed to be static. The simulated signals are processed using a modified version of the GSNRx ${ }^{\mathrm{TM}}$ software receiver [26] to generate code and carrier phase measurements. The software receiver was modified to incorporate multi-antenna processing with Power Minimization (PM) and MPDR processing techniques.

\subsubsection{Power Minimization (PM)}

The PM approach was implemented in the pre-correlation stage with the receiver architecture shown in Figure 2. The covariance matrix of the received signal can be obtained as

$$
\mathbf{R}_{\mathbf{x x}}=\frac{1}{T} \sum_{1}^{T} \mathbf{x x}^{H}
$$

where $T$ is the number of temporal Intermediate Frequency (IF) samples and $\mathbf{R}_{\mathbf{x x}}$ is of dimension $M N \times M N$. 
The optimum weight vector is given by [27]

$$
\mathbf{w}_{\mathrm{PM}}=\frac{\mathbf{R}_{\mathbf{x x}}^{-1} \mathbf{q}}{\mathbf{q}^{H} \mathbf{R}_{\mathbf{x x}}^{-1} \mathbf{q}}
$$

where $\mathbf{q}$ is the constraint vector with dimension $M N \times 1$ and given by

$$
\mathbf{q}=[1,0,0, \ldots, 0]^{T}
$$

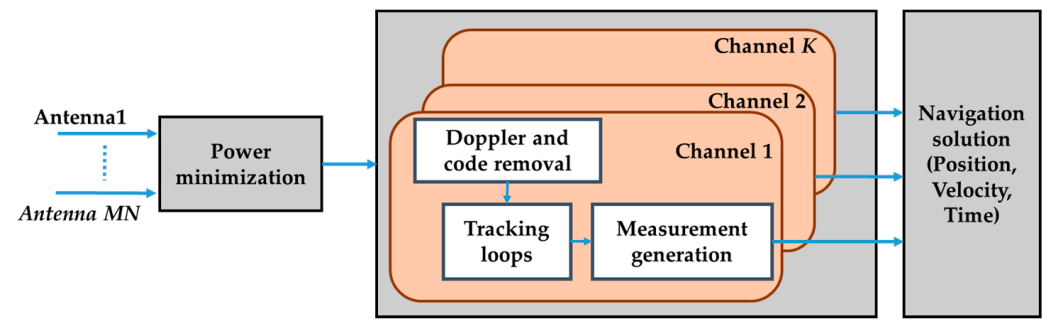

Figure 2. Multi-antenna processing using PM beamforming.

\subsubsection{Minimum Power Distortionless Response (MPDR)}

MPDR was performed at the post-correlation stage with the MPDR receiver architecture shown in Figure 3. IF signals from all the antennas are fed to each tracking channel. In each tracking channel, the code and carrier replica signals from the beamformed data are used to de-spread other antenna signals in order to maintain relative phase differences between the antenna elements. The optimum weight vector for the MPDR beamformer for the $k$ th satellite is given by [24]

$$
\mathbf{w}_{\mathrm{PM}}=\frac{\mathbf{R}_{\mathbf{x x}}^{-1} \mathbf{a}_{k}}{\mathbf{a}_{k}^{H} \mathbf{R}_{\mathbf{x x}}^{-1} \mathbf{a}_{k}}
$$

The covariance matrix $\mathbf{R}_{\mathbf{x x}}$ was computed at the pre-correlation stage because at this stage, interference power might be reduced due to the correlation process affecting beamformer performance. The combined correlator outputs will be used to generate code and carrier phase measurements.

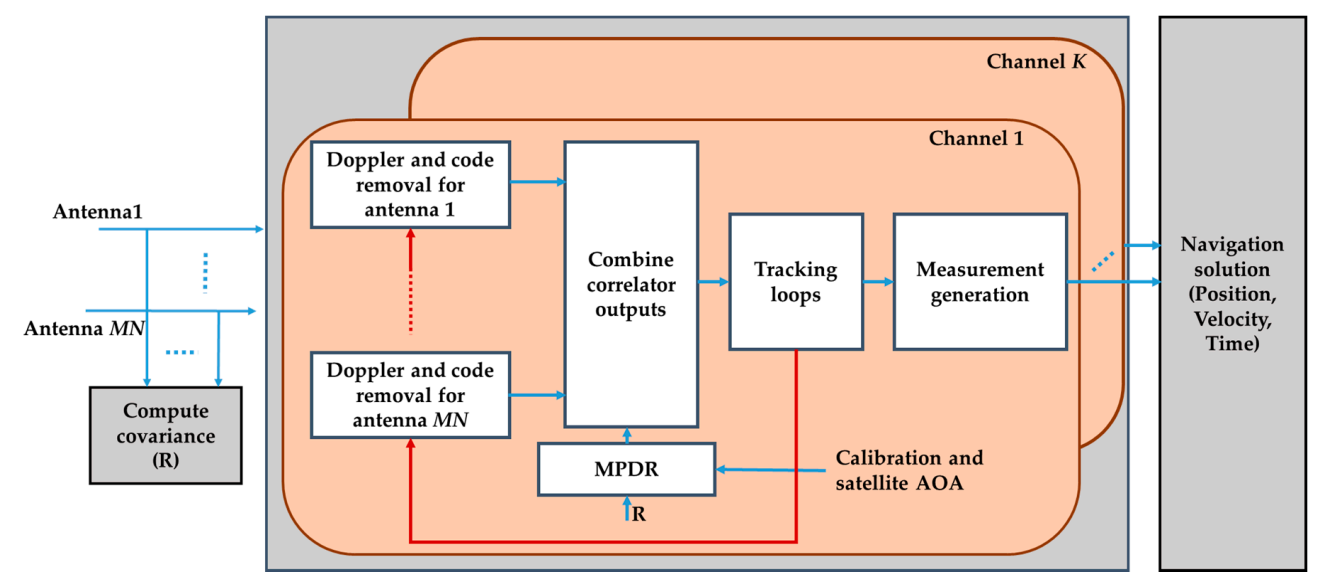

Figure 3. Multi-antenna processing approach using MPDR beamforming.

\subsection{Measurement Distortions Analysis}

After interference suppression, the main goal is to characterize distortions in terms of mean and standard deviations in the code and carrier phase measurements from the beamforming process in 
the presence of interference. A differential technique is used to evaluate the measurement distortions. Measurements from one of the array antennas with clean data without interference acts as a reference channel. Single differencing between reference antenna measurements (with clean data) and those from the beamformed data (in the presence of interference) acts as a zero-baseline test. The resulting single difference should ideally have a zero mean if there are no distortions in the measurements from the antenna array processing. Measurement distortions refer to the distortions caused by the multi-antenna GNSS receiver process in the presence of interference.

\subsubsection{Pseudorange Measurements Analysis}

The pseudorange measurement of the $k$ th satellite after beamforming in the presence of interference can be expressed as

$$
P_{\text {array }}^{k}=\rho^{k}+d \rho^{k}+c\left(d t^{k}-d T\right)+d_{\text {iono }}^{k}+d_{\text {tropo }}^{k}+\rho_{\text {bias }}^{k}+\varepsilon_{\rho}^{k}
$$

where $\rho^{k}$ is the true range, $d \rho^{k}$ is the orbital error, $d t^{k}$ and $d T$ are the satellite and receiver clock errors, $d_{\text {iono }}^{k}$ is the ionosphere error, $d_{\text {tropo }}^{k}$ is the tropospheric delay, $\varepsilon_{\rho}^{k}$ is the receiver code noise, and $\rho_{\text {bias }}^{k}$ is the possible bias introduced by antenna array processing.

The pseudorange from the $k$ th satellite for the reference antenna without interference can be expressed as

$$
P_{r e f}^{k}=\rho^{k}+d \rho^{k}+c\left(d t^{k}-d T\right)+d_{i o n o}^{k}+d_{\text {tropo }}^{k}+\varepsilon_{\rho}^{k}
$$

The single difference between the pseudorange measurements obtained from the reference antenna and after beamforming is

$$
\Delta P^{k}=P_{r e f}^{k}-P_{\text {array }}^{k}=\rho_{\text {bias }}^{k}+\varepsilon_{\Delta \rho}^{k}
$$

where $\varepsilon_{\Delta \rho}^{k}$ is the single differenced receiver code noise.

Since this single differencing acts as a zero-baseline, the atmospheric error and multipath cancel out. The code noise standard deviation depends on receiver tracking strategies such as chip spacing and the discriminators used and it should ideally be zero mean. Hence, one can estimate the pseudorange bias induced by the antenna array processing in the presence of interference. It is assumed that the residual multipath effect is small compared to the antenna array induced biases. In the simulations, GPS signals were simulated in multipath free conditions. In the case of actual data collection, a low multipath environment was selected.

\subsubsection{Carrier Phase Measurements Analysis}

Similar to pseudorange measurement bias estimation, the carrier phase measurement bias can also be estimated by performing single differencing between the reference antenna and beamformed carrier phase measurements.

The carrier phase measurements of the $k$ th satellite after beamforming in the presence of interference can be expressed as

$$
\phi_{\text {array }}^{k}=\rho^{k}+d \rho^{k}+c\left(d t^{k}-d T\right)+\lambda N^{k}-d_{\text {iono }}^{k}+d_{\text {tropo }}^{k}+\phi_{\text {bias }}^{k}+\varepsilon_{\phi}^{k}
$$

where $\varepsilon_{\phi}^{k}$ is the receiver carrier phase noise, $N^{k}$ is the integer ambiguity, and $\phi_{b i a s}^{k}$ is the possible carrier phase bias introduced by antenna array processing.

The carrier phase measurement from the $k$ th satellite for the reference antenna without interference can be expressed as

$$
\phi_{\text {ref }}^{k}=\rho^{k}+d \rho^{k}+c\left(d t^{k}-d T\right)+\lambda N^{k}-d_{\text {iono }}^{k}+d_{\text {tropo }}^{k}+\varepsilon_{\phi}^{k}
$$


The single difference between the pseudorange measurements obtained from the reference antenna and after beamforming is

$$
\Delta \phi^{k}=\phi_{\text {ref }}^{k}-\phi_{\text {array }}^{k}=\phi_{\text {bias }}^{k}+\varepsilon_{\Delta \phi}^{k}
$$

where $\varepsilon_{\Delta \phi}^{k}$ is the single differenced receiver carrier phase noise.

After single differencing, atmospheric errors cancel out. There is no receiver clock error since a single receiver is used. Integer ambiguity is also removed since a coherent-phase multi-channel front-end using a single oscillator is used which makes receiver to initialize ambiguity to the same value for the single channel and multi-antenna receiver process. As in the pseudorange case, carrier phase noise is ideally zero mean and therefore any bias observed using single differencing can be attributed to array processing in the presence of interference.

As this research focuses on high precision applications, antennas with good phase centre stability and low mutual coupling are considered. Hence, the effect of phase centre and mutual coupling are not considered in the pseudorange and carrier phase measurement formulations (Equations (17)-(22)).

\section{Simulation Results and Discussion}

The effect of different types of interference sources and array processing approaches are now studied through simulations. Simulations were carried out considering different interference types. In order to evaluate the effect of different kinds of interference and different beamforming methods, it is assumed that only one interference source is present at the time in each simulation scenario. The interference sources considered are Continuous Wave (CW) interference, chirp interference, and band-limited additive white Gaussian noise. The effect of these interference sources on GNSS receiver operation differs due to their spectral characteristics. Therefore, it is of interest to check the performance of the array based receiver under different interference types. Even though the power and the number of interference sources eventually affect beamformer performance, the main goal here is to compare PM and MPDR beamformers for high precision applications.

The input IF data file for the multi-antenna software simulator was collected using a Spirent hardware GPS simulator [28] by disabling multipath and atmospheric errors. A static user scenario was used for collecting IF data samples at a $5 \mathrm{MHz}$ sampling rate. The multi-antenna GPS signals were generated using the multi-antenna signal simulator and interference was added later to the clean IF samples. The six-element rectangular antenna array described earlier was considered for the simulations. The interference source was assumed to be static and was added after $15 \mathrm{~s}$ in order for the receiver to acquire and track the signals (for the post-correlation beamforming). The multi-antenna signal simulation method and sky plot of the simulated GPS satellites and interference source direction are shown in Figure 4. The characteristics of the interference source are given in Table 1. In all the simulation scenarios, the interference source direction was the same. The $\mathrm{C} / \mathrm{N}_{0}$ values for all PRNs were set to be the same for all the antenna elements ( $48 \mathrm{~dB}-\mathrm{Hz})$.

Table 1. Interference sources considered in the simulations and their characteristics.

\begin{tabular}{cll}
\hline Simulation Scenarios & Interference Type & \multicolumn{1}{c}{ Interference Characteristics } \\
\hline Scenario 1 & CW interference & $\begin{array}{l}\text { The frequency of the CW interference is } 500 \mathrm{~Hz} \text { away from IF } \\
(420 \mathrm{kHz}) . \text { Jammer-to-signal power is assumed to be } 30 \mathrm{~dB} .\end{array}$ \\
Scenario 2 & Chirp interference & $\begin{array}{l}\text { Chirp signal bandwidth is } 11.8 \mathrm{MHz} \text { with an interval of } \\
11.7 \mu \mathrm{s} . \text { These values are basically chosen from commercially } \\
\text { available in-car jammers. Jammer-to-signal power is 30 dB. }\end{array}$ \\
Scenario 3 & Band limited white \\
Gaussian noise & $\begin{array}{l}\text { White Gaussian noise is generated through the MATLAB } \\
\text { awgn() function with a bandwidth of } 5 \mathrm{MHz} \text { and } \\
\text { jammer-to-signal power of 30 dB. }\end{array}$ \\
\hline
\end{tabular}



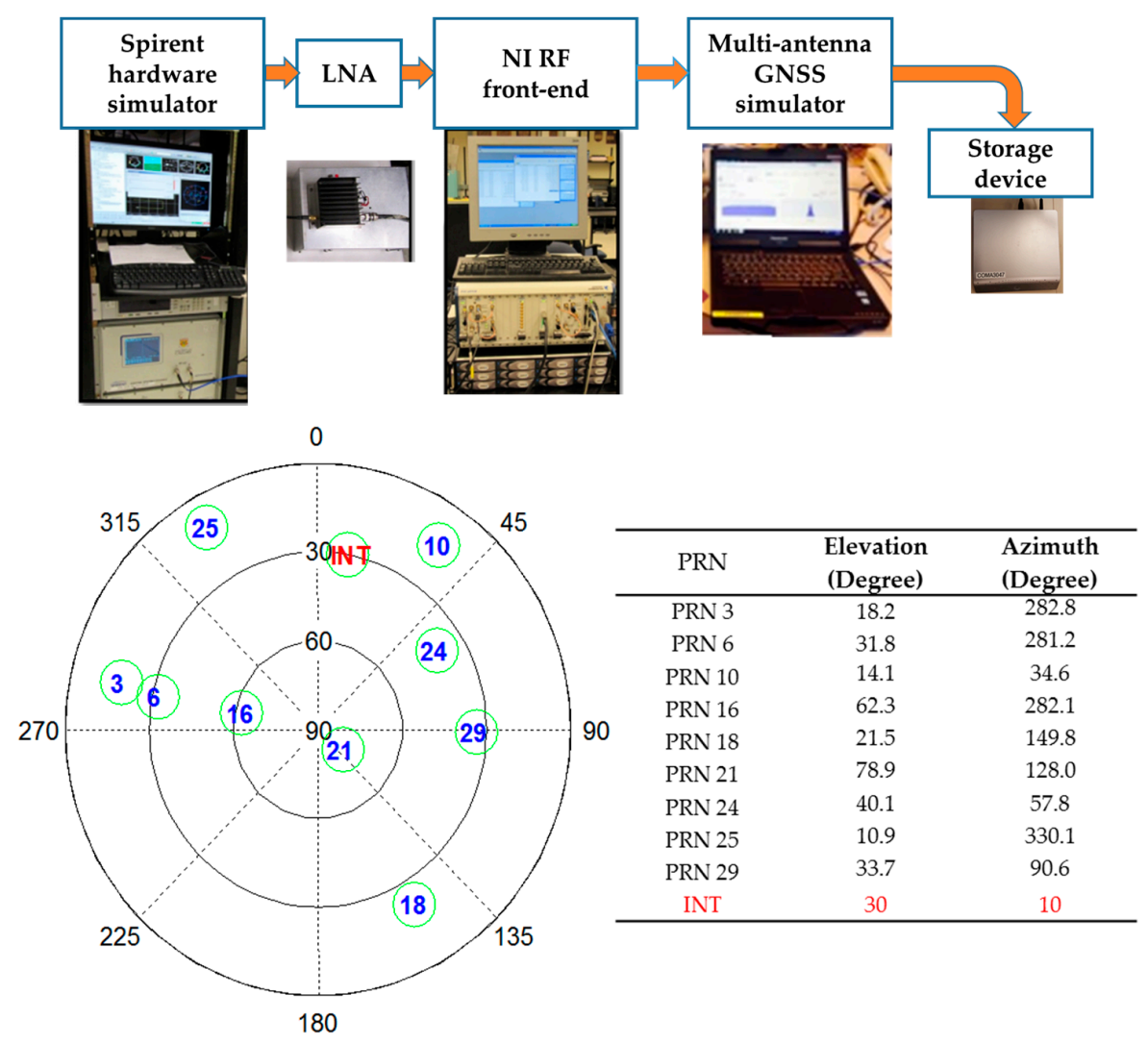

Figure 4. Multi-antenna signal simulation set-up and sky plot of the simulated GPS satellites and interference (INT) source.

The simulated signals were processed using GSNRx ${ }^{\mathrm{TM}}$ with the architectures shown in Figures 2 and 3 to generate tracking and measurement parameters. Here, PM was performed from the beginning of the dataset as it was done in the pre-correlation stage. In the case of the MPDR beamformer, the receiver was made to operate with a single antenna for the initial $10 \mathrm{~s}$, after which beamforming was performed before the occurrence of interference. This was done to ensure that the receiver operates in beamforming mode when interference affects the IF samples. In both PM and MPDR approaches, correlator spacing of 0.4 between early and late arms and non-coherent early-minus late discriminators were used. Carrier tracking was performed using a third order Phase Locked Loop (PLL) with a $15 \mathrm{~Hz}$ bandwidth.

\subsection{Tracking Domain Analysis}

In this section, tracking performance in terms of carrier Doppler estimation, $\mathrm{C} / \mathrm{N}_{0}$ and Phase Lock Indicator (PLI) is evaluated. The $\mathrm{C} / \mathrm{N}_{0}$ is computed using narrow band power and wide band power of the prompt correlation values and PLI is computed using prompt in-phase and quadrature-phase values [29]. Initially, the signal affected by interference from the reference antenna of the array was processed and the corresponding results are discussed in subsequent sections. Since PRN 6 and PRN 16 are more affected by CW interference, these two PRNs are used to analyze tracking performance of the array-based receiver.

The tracking outputs of PRN 6 for the single antenna case affected by CW interference after beamforming for the first $20 \mathrm{~s}$ are shown in Figure 5 where 'Single Antenna' corresponds to the tracking outputs of the reference antenna of the array in the presence of interference. For the initial $15 \mathrm{~s}$, there was no interference; as mentioned earlier, PM was performed from the beginning of the data and MPDR was performed after $10 \mathrm{~s}$. 

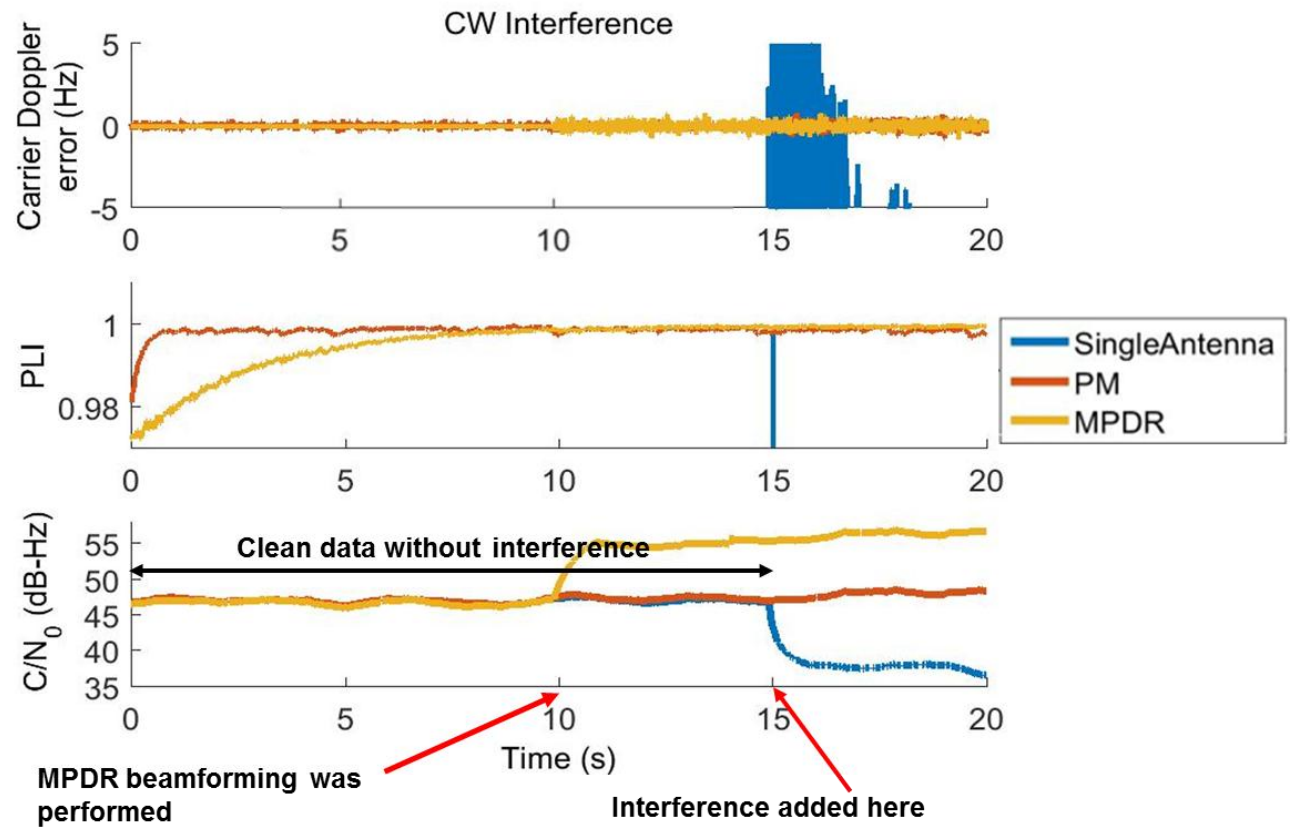

Figure 5. Tracking performance of PRN 6 with $\mathrm{CW}$ interference.

Let us first consider the initial $15 \mathrm{~s}$ of data without interference. After MPDR beamforming, a $\mathrm{C} / \mathrm{N}_{0}$ gain of $7.5 \mathrm{~dB}$ is observed. As six antenna elements were used, a gain of $7.7 \mathrm{~dB}$ [10 $\times \log _{10}$ (number of antennas)] is expected and one can observe the same with MPDR beamforming. With PM, a gain of $1.5 \mathrm{~dB}$ is observed; in this case, since there is no constraint on for the satellite signal AOA, the gain depends on the beampattern, which in turn depends on the interference direction and array geometry.

Now consider the data affected by interference (after $15 \mathrm{~s}$ ) in Figure 5. As soon as interference is injected, the $C / N_{0}$ values of the single antenna drops by about $8 \mathrm{~dB}$. However, the same $C / N_{0}$ as that without interference was maintained through MPDR and PM methods. This ensured successful mitigation of the interference. If one considers the $\mathrm{C} / \mathrm{N}_{0}$ gain in the presence of interference, MPDR was able to deliver nearly $15.5 \mathrm{~dB}$ with respect to the single antenna. The gain observed from PM was $9.5 \mathrm{~dB}$. As shown in Figure 5, Doppler errors increase and PLI degrades for the single antenna case after interference. However, MPDR and PM were able to provide the same performance as that for the interference-free case. To compute the carrier Doppler errors shown in Figure 5, the Doppler values obtained from the reference antenna with clean data were used as reference.

The carrier tracking performance of PRN 6 for chirp and BWGN scenarios for the first $60 \mathrm{~s}$ are shown in Figures 6 and 7. MPDR was able to provide $15.5 \mathrm{~dB}$ of gain even in the presence of chirp and BWGN interference. PM was able to provide $9.5 \mathrm{~dB}$ of gain in the presence of the same interference. The Doppler error variance is lower after beamforming; this can be clearly observed in Figure 7 in the presence of BWGN interference. The receiver was able to maintain PLI values of 0.99 after beamforming in all interference scenarios, indicating reliable tracking of the carrier phase.

The performance results of different PRNs in terms of Root-Mean-Square (RMS) Doppler errors, $\mathrm{C} / \mathrm{N}_{0}$ gain, and mean PLI for chirp interference are listed in Table 2. These values were computed using the tracking outputs in the presence of interference. For computing Root-Mean-Square Error (RMSE) Doppler errors, the Doppler values obtained from the reference antenna with clean data were used as true values. The tracking performance results of different PRNs in the presence of CW and BWGN jammers are provided in Appendix A for the CW jammer in Table A1 and those for BWGN in Table A2. It can be observed that compared to single antenna results, RMS Doppler errors are reduced for both beamformers for all simulation scenarios. MPDR RMS Doppler errors were lower by $0.1 \mathrm{~Hz}$ as compared to PM, a relative improvement of $25 \%$. 

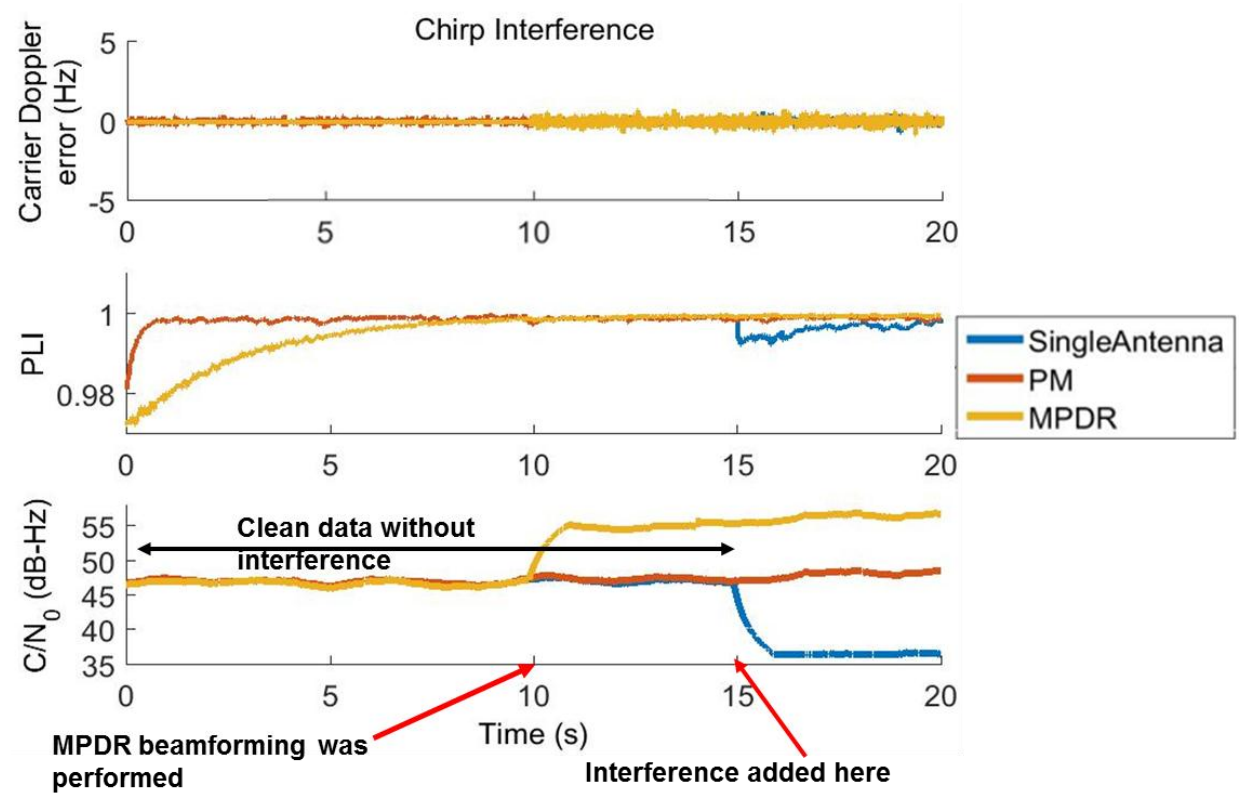

Figure 6. Carrier tracking performance of PRN 6 with chirp interference.
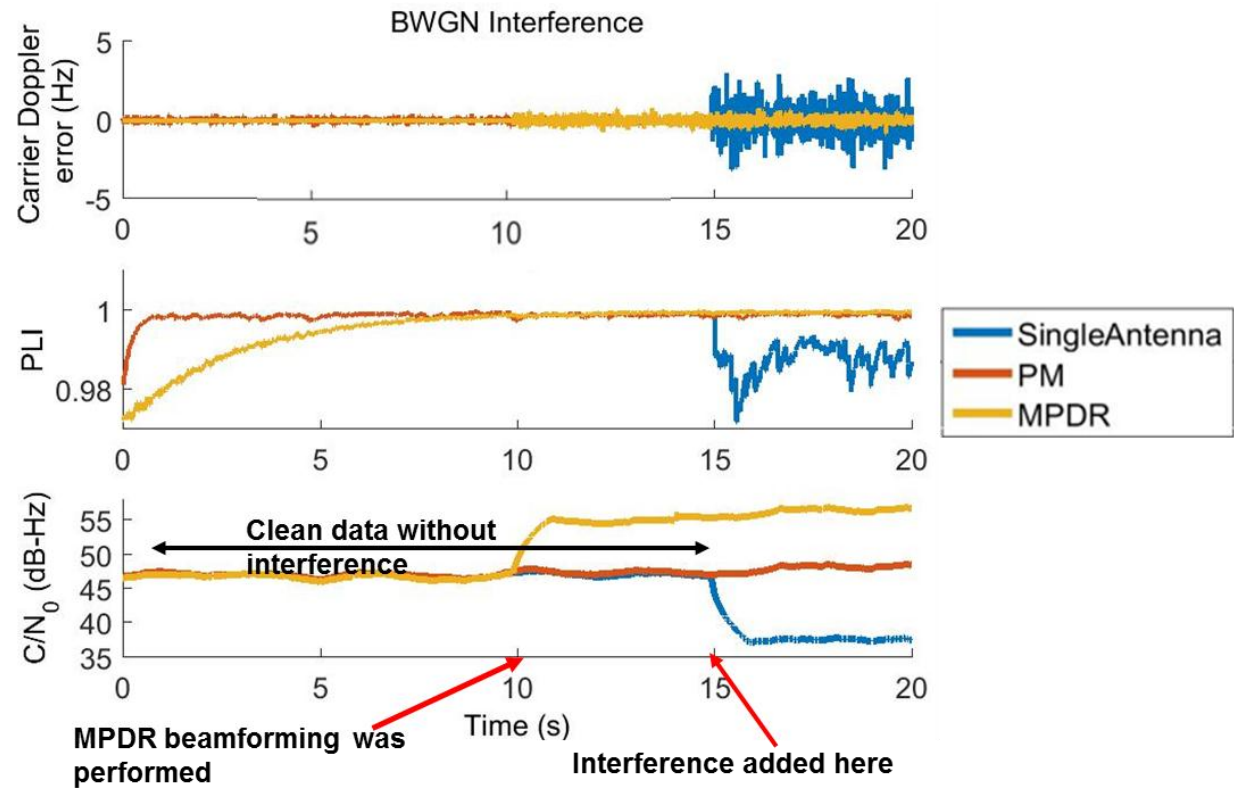

Figure 7. Carrier tracking performance of PRN 6 with Band limited white Gaussian noise.

Table 2. Tracking performance of different PRNs in the presence of chirp interference.

\begin{tabular}{|c|c|c|c|c|c|c|c|c|c|}
\hline \multirow[b]{2}{*}{ PRN } & \multicolumn{3}{|c|}{ Single Antenna } & \multicolumn{3}{|c|}{ PM } & \multicolumn{3}{|c|}{ MPDR } \\
\hline & $\begin{array}{l}\text { RMSE Doppler } \\
\text { (Hz) }\end{array}$ & PLI & $\begin{array}{c}\mathrm{C} / \mathrm{N}_{0} \\
\text { (dB-Hz) }\end{array}$ & $\begin{array}{l}\text { RMSE Doppler } \\
\text { (Hz) }\end{array}$ & PLI & $\underset{\text { (dB-Hz) }}{\mathrm{C} / \mathrm{N}_{0}}$ & $\begin{array}{l}\text { RMSE Doppler } \\
\text { (Hz) }\end{array}$ & PLI & $\begin{array}{c}\mathrm{C} / \mathrm{N}_{0} \\
\text { (dB-Hz) }\end{array}$ \\
\hline PRN 3 & 0.45 & 0.99 & 36.6 & 0.32 & 0.99 & 48.9 & 0.26 & 0.99 & 54.9 \\
\hline PRN 6 & 0.40 & 0.99 & 36.6 & 0.36 & 0.99 & 48.0 & 0.28 & 0.99 & 54.7 \\
\hline PRN 10 & 0.46 & 0.99 & 36.7 & 0.78 & 0.99 & 45.8 & 0.36 & 0.99 & 54.4 \\
\hline PRN 16 & 0.74 & 0.99 & 36.7 & 0.61 & 0.99 & 45.9 & 0.51 & 0.99 & 54.4 \\
\hline PRN 18 & 0.43 & 0.99 & 36.7 & 0.40 & 0.99 & 47.3 & 0.35 & 0.99 & 54.4 \\
\hline PRN 21 & 0.42 & 0.99 & 36.7 & 0.35 & 0.99 & 48.5 & 0.33 & 0.99 & 54.8 \\
\hline PRN 24 & 0.48 & 0.99 & 36.6 & 0.46 & 0.99 & 47.6 & 0.36 & 0.99 & 54.0 \\
\hline PRN 25 & 0.47 & 0.99 & 36.7 & 0.43 & 0.99 & 47.0 & 0.33 & 0.99 & 54.9 \\
\hline PRN 29 & 0.55 & 0.99 & 36.7 & 0.49 & 0.99 & 45.4 & 0.46 & 0.99 & 54.4 \\
\hline
\end{tabular}




\subsection{Measurement Domain Analysis}

This section analyzes pseudorange and carrier phase measurement errors after beamforming. The measurement domain results in the presence of chirp interference are provided here. The measurement differencing technique described in the methodology section is used to obtain pseudorange and carrier phase measurement errors.

A time series plot of the pseudorange and carrier phase measurement errors for both PM and MPDR are shown in Figure 8. The mean and standard deviations of the pseudorange errors for all the PRNs are provided in Table 3. First consider the results of PM. It can be seen from Table 3 that PM induces pseudorange biases of the order of $30 \mathrm{~cm}$ to $50 \mathrm{~cm}$ for different PRNs. It can also be observed that the biases observed are different for different PRNs. It can be seen from Figure 8 that PRN 10 distortions are higher than those of other PRNs. This is because the interference source AOA is close to that of PRN 10 as shown in the sky plot of Figure 4. Now consider the MPDR beamformer; it induces up to $4 \mathrm{~cm}$ of pseudorange bias, which is about the code phase measurement noise. For PRN 10, which is near the interference source, standard deviations of the measurement errors have increased compared to other PRNs.
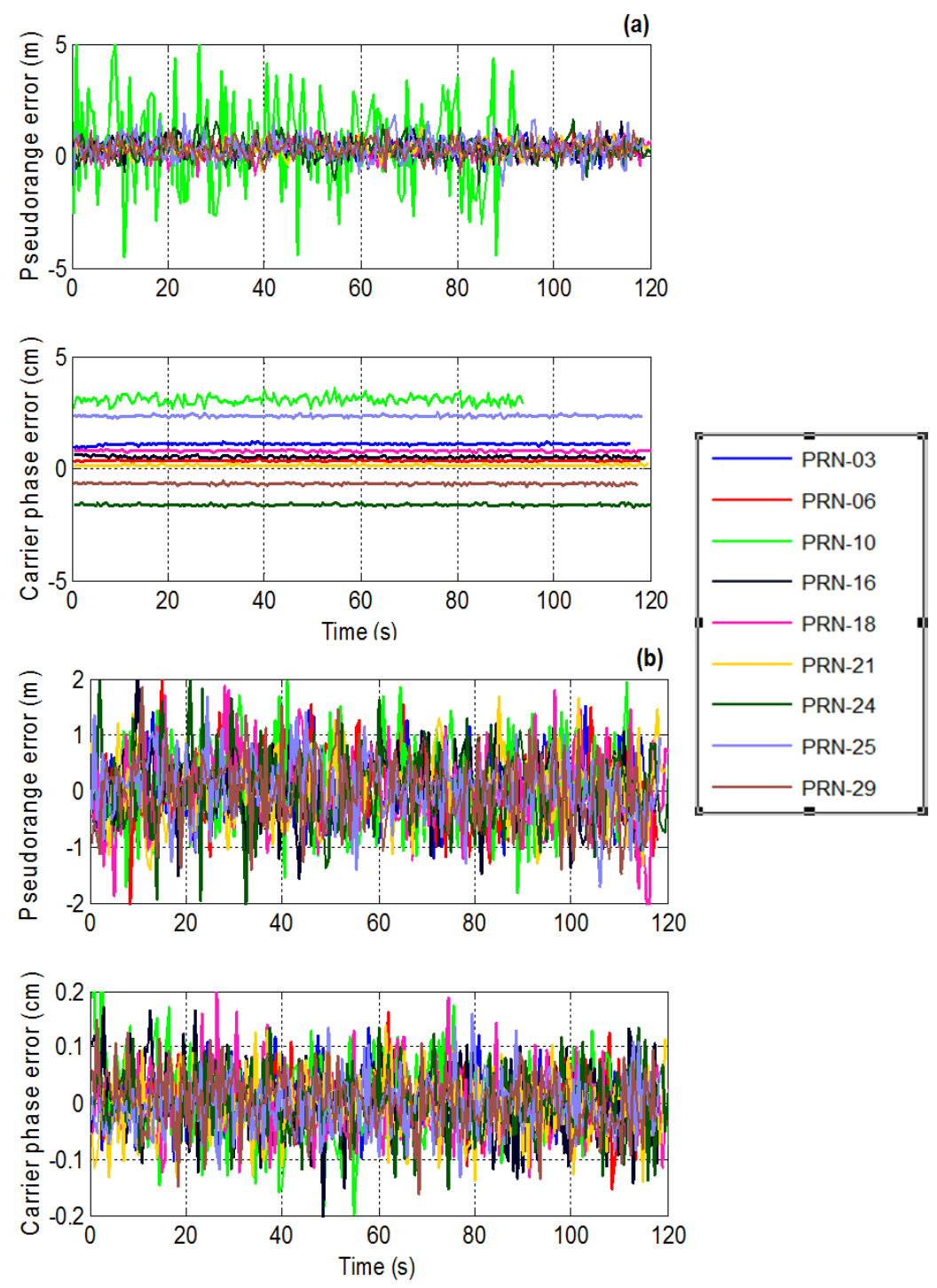

Figure 8. Time series plot of the pseudorange and carrier phase measurement errors using (a) PM; (b) MPDR. 
Table 3. Pseudorange measurement errors for PM and MPDR in the presence of chirp interference.

\begin{tabular}{cccccc}
\hline \multirow{2}{*}{ PRN } & \multicolumn{2}{c}{ PM } & & \multicolumn{2}{c}{ MPDR } \\
\cline { 2 - 3 } \cline { 5 - 5 } \cline { 5 - 5 } & Mean $(\mathbf{m})$ & Standard Deviation $(\mathbf{m})$ & & Mean $(\mathbf{m})$ & Standard Deviation $(\mathbf{m})$ \\
\hline PRN 3 & 0.27 & 0.31 & 0.02 & 0.55 \\
PRN 6 & 0.33 & 0.27 & 0.02 & 0.59 \\
PRN 10 & 0.50 & 1.93 & 0.08 & 0.75 \\
PRN 16 & 0.31 & 0.36 & & 0.01 & 0.59 \\
PRN 18 & 0.28 & 0.33 & & 0.02 & 0.69 \\
PRN 21 & 0.31 & 0.30 & -0.04 & 0.58 \\
PRN 24 & 0.35 & 0.49 & 0.02 & 0.65 \\
PRN 25 & 0.38 & 0.55 & 0.04 & 0.55 \\
PRN 29 & 0.31 & 0.40 & -0.01 & 0.60 \\
\hline
\end{tabular}

It can be observed that mean pseudorange bias values are higher for PM than MPDR. The mean and standard deviations of the carrier phase measurement errors for different PRNs are listed in Table 4. It can be observed from Figure 8 that PM induces different biases for different PRNs. The bias for a particular PRN remains the same for the entire test duration. This is because the interference source location and the array orientation were fixed and as such the beamformer weights were constant during the test. However, if the interference direction is changed or in the case of a dynamic user, the beamformer weights also change, different biases might be observed for the same PRN. A maximum bias value of $30 \mathrm{~mm}$ was observed in PRN 10 bias in the case of the PM beamformer. The carrier phase measurement biases for the MPDR beamformer are minimal as listed in Table 4.

Table 4. Carrier phase measurement errors using PM and MPDR in the presence of chirp interference.

\begin{tabular}{cccccc}
\hline \multirow{2}{*}{ PRN } & \multicolumn{2}{c}{ PM } & & \multicolumn{2}{c}{ MPDR } \\
\cline { 2 - 3 } \cline { 5 - 6 } \cline { 5 - 6 } & Mean $(\mathbf{m m})$ & Standard Deviation $(\mathbf{m m})$ & & Mean $(\mathbf{m m})$ & Standard Deviation $(\mathbf{m m})$ \\
\hline PRN 3 & 10.7 & 0.4 & 0.04 & 0.4 \\
PRN 6 & 3.4 & 0.2 & -0.01 & 0.4 \\
PRN 10 & 30.5 & 1.8 & & 0.07 & 0.7 \\
PRN 16 & 5.0 & 0.4 & & 0.05 & 0.6 \\
PRN 18 & 7.6 & 0.3 & -0.01 & 0.5 \\
PRN 21 & 1.4 & 0.2 & -0.05 & 0.5 \\
PRN 24 & -16.2 & 0.4 & & 0.01 & 0.5 \\
PRN 25 & 23.3 & 0.4 & -0.03 & 0.5 \\
PRN 29 & -6.9 & 0.3 & 0.03 & 0.5 \\
\hline
\end{tabular}

Similar observations were made with CW and BWGN jammers for code and carrier phase measurement distortions. The results corresponding to these jammers are provided in Tables B1-B4.

\subsection{Position Domain Analysis}

For the position domain analysis, the code and carrier phase measurements were processed using the RTKLib open source software [30]. The measurements generated using the reference antenna of the array without interference were used as the base observations for the RTK software. The remote observations were generated from the beamformed data affected by interference. This kind of processing acts like a zero-baseline and any distortion in the measurements affects the position solution. The open source software was used without any modifications. In the RTKLib, the continuous carrier phase ambiguity resolution mode was selected and forward-backward processing was performed.

The east, north, and height position errors for PM and MPDR with chirp interference are shown in Figure 9. The RTK software outputs a quality flag for each solution update indicating a fixed or float solution. PM was not able to provide fixed solutions and position errors on the order of $10 \mathrm{~cm}$ occur. MPDR was able to provide fixed solutions and position errors were at the sub-millimetre level. 
The mean and standard deviations of the position errors observed for both beamformers are provided in Table 5. In the PM case, satellite directions are not considered in the optimization criterion and the antenna phase values are not added coherently. This will have adverse effect on the carrier phase measurements as discussed in the measurement analysis section. These biased measurements will affect carrier phase based positioning. It should be noted that with PM, different biases are observed for different PRNs and these bias values are a function of the interference and satellites AOA and cannot be calibrated beforehand.
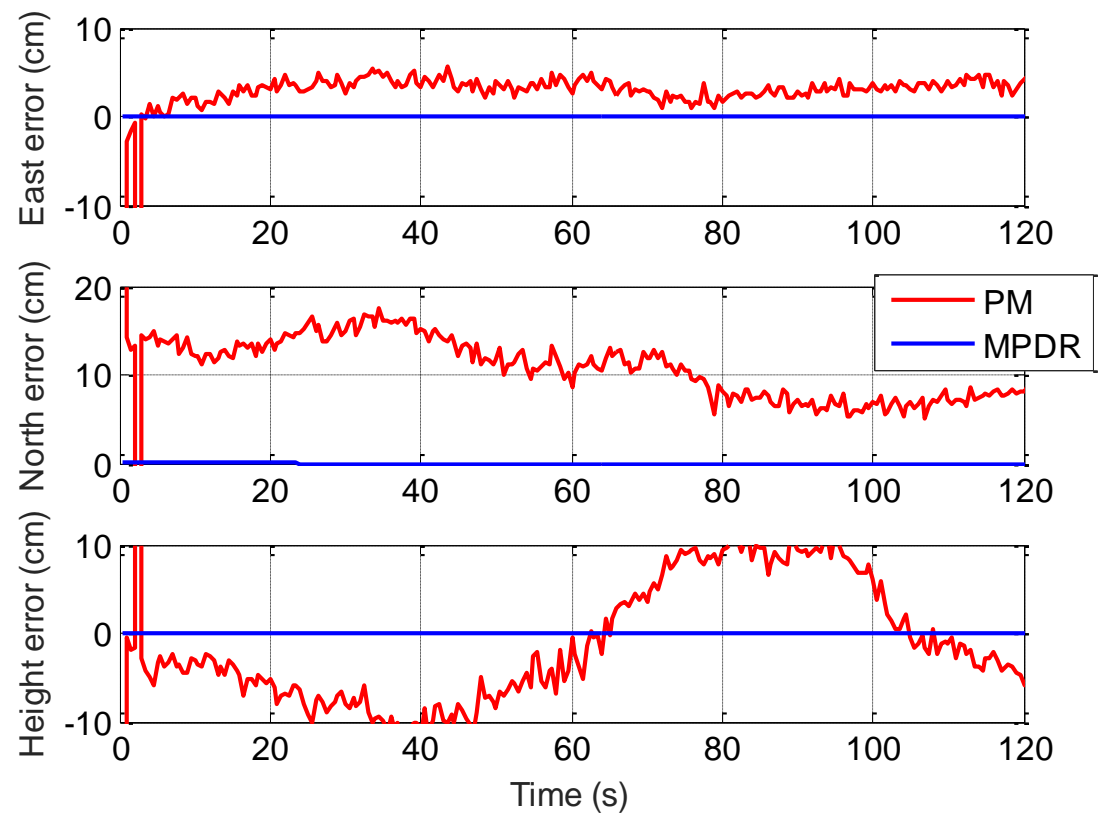

Figure 9. Carrier phase position errors with PM and MPDR in the presence of chirp interference (Processed using RTKLib open source software).

Table 5. Carrier phase position errors with PM and MPDR.

\begin{tabular}{cccccc}
\hline \multirow{2}{*}{ Position Errors } & \multicolumn{2}{c}{ PM } & & \multicolumn{2}{c}{ MPDR } \\
\cline { 2 - 3 } \cline { 5 - 6 } & Mean & Standard Deviation & & Mean & Standard Deviation \\
\hline East error $(\mathrm{cm})$ & 3.2 & 1.1 & & -0.001 & 0.003 \\
North error $(\mathrm{cm})$ & 10.6 & 3.3 & & 0.002 & 0.005 \\
Height error $(\mathrm{cm})$ & -1.4 & 6.8 & & -0.005 & 0.005 \\
\hline
\end{tabular}

\subsection{Effect of Interference AOA on Measurements}

The relative direction between jammer and satellite signals plays a critical role in anti-jam applications using antenna array processing. The effect of changing the AOA of the interference source is therefore analyzed. It is assumed that only one chirp interference source is present. Time series plot of the pseudorange and carrier phase measurements in the presence of chirp interference for PM and MPDR are shown in Figure 10. The interference direction is changed after $65 \mathrm{~s}$. For the initial $65 \mathrm{~s}$, the interference is located at (elevation $=30^{\circ}$, azimuth $=10^{\circ}$ ) and then changed to (elevation $=30^{\circ}$, azimuth $=225^{\circ}$ ). It can be observed that the carrier phase biases observed in PM changes when the interference direction changes. However, MPDR beamformer does not induce any biases in the carrier phase measurements, irrespective of the AOA of the interference. For the initial $65 \mathrm{~s}$, interference location was near PRN 10 and due to this, the standard deviation of the pseudorange measurements for PRN 10 was high. After 65 s, the interference direction was away from PRN 10 and the standard deviation of the pseudorange measurements decreased. 

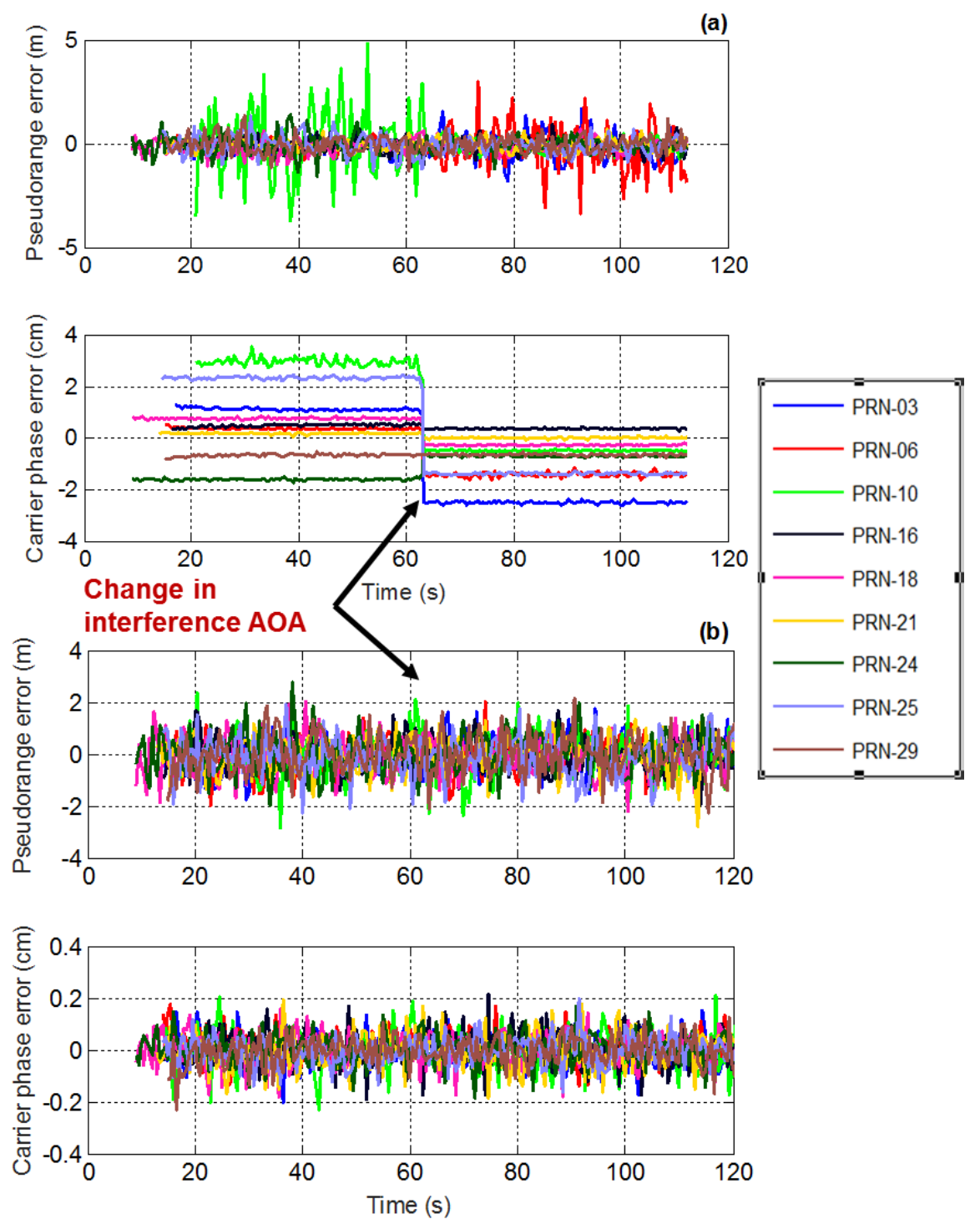

Figure 10. Effect of change in AOA of interference on pseudorange and carrier phase measurements for (a) PM; and (b) MPDR.

\section{Field-Test Results and Discussion}

This section describes field test results using PM and MPDR beamforming. Unlike the simulation case, the data collected in an open sky condition was subject to actual antenna array errors such as antenna phase centre instability, phase and gain mismatch between different receiver channels, and mutual coupling. A rectangular array with six antenna elements shown in Figure 11 was used for the data collection. Novatel 501 antenna elements [31] were used to construct the array. The setup used and sky plot of the visible GPS satellites are shown in Figure 11. A NovAtel Synchronous Position, Attitude and Navigation (SPAN) LCI mounted on the array platform was used to obtain the array attitude used for calibration and MPDR beamforming. A Fraunhofer multi-antenna RF front-end was used to collect IF samples from all the six antennas simultaneously in an open sky environment and low multipath conditions. The initial few seconds of the data were used to perform antenna array calibration. Interference was added to the data through a MATLAB ${ }^{\mathrm{TM}}$ software script. Although interference was added later to the actual data as in the case of simulations, the difference between these simulations and field tests is the calibration of an actual array and mutual coupling which might affect the performance of the beamformer. The interference source is assumed to be static with 
a jammer-to-signal power of $30 \mathrm{~dB}$. As beamformer performance was similar for different types of jammers, results corresponding to the chip jammer are provided in the field test results.
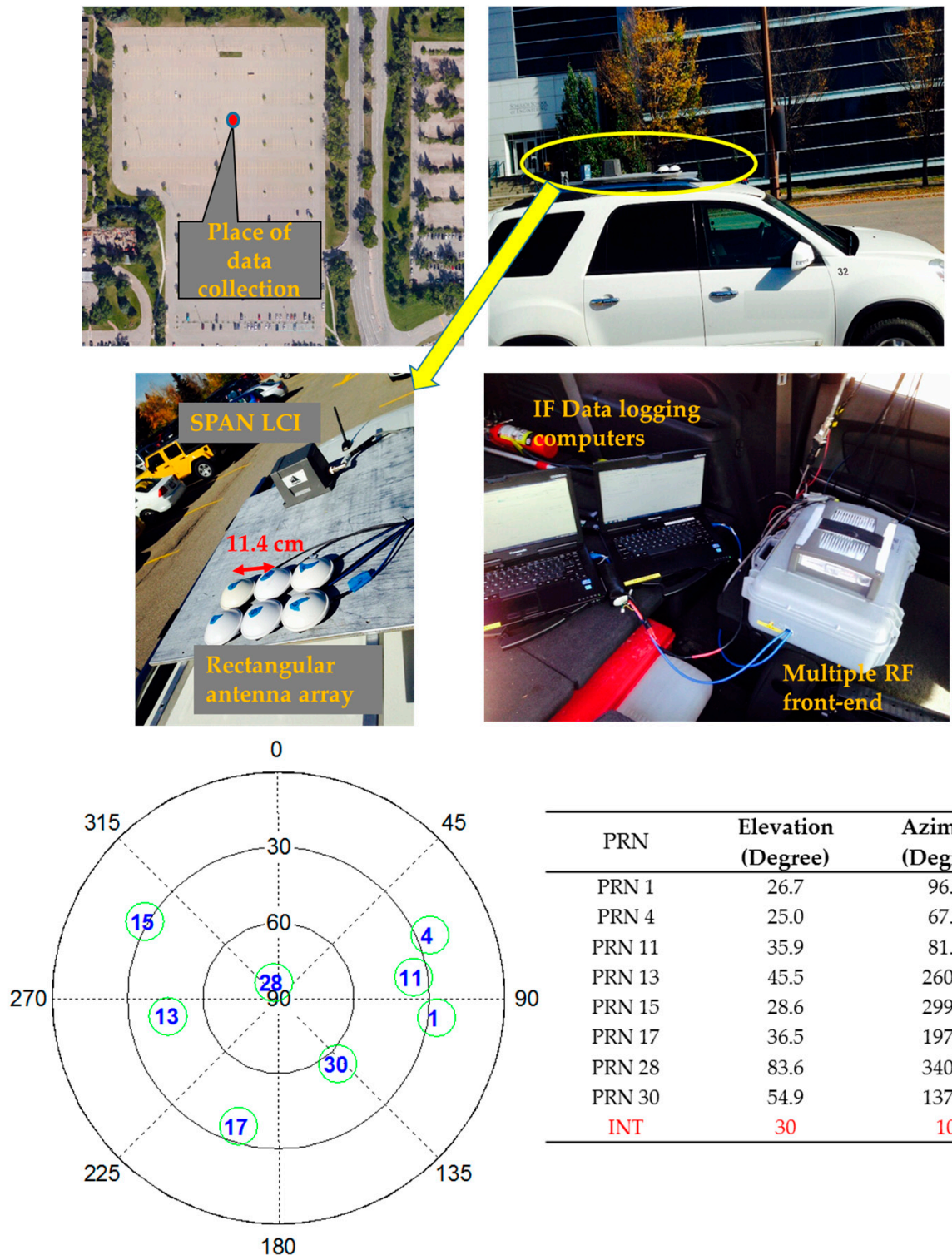

\begin{tabular}{ccc}
\hline PRN & $\begin{array}{c}\text { Elevation } \\
\text { (Degree) }\end{array}$ & $\begin{array}{c}\text { Azimuth } \\
\text { (Degree) }\end{array}$ \\
\hline PRN 1 & 26.7 & 96.6 \\
PRN 4 & 25.0 & 67.3 \\
PRN 11 & 35.9 & 81.3 \\
PRN 13 & 45.5 & 260.7 \\
PRN 15 & 28.6 & 299.9 \\
PRN 17 & 36.5 & 197.5 \\
PRN 28 & 83.6 & 340.8 \\
PRN 30 & 54.9 & 137.9 \\
INT & 30 & 10 \\
\hline
\end{tabular}

Figure 11. Field data collection setup and sky plot of visible GPS satellites.

The collected data without interference and after adding interference were processed with $\mathrm{GSNRx}^{\mathrm{TM}}$ to generate tracking parameters and measurements. A correlator spacing of 0.2 between the early and late arms and non-coherent early-minus late discriminator was used. Carrier tracking was performed using a third order PLL with a $15 \mathrm{~Hz}$ bandwidth.

\subsection{Tracking Domain Analysis}

The PM beamforming was performed from the beginning of the data and MPDR was performed after $10 \mathrm{~s}$. The interference was added to the data after $15 \mathrm{~s}$. Tracking performance of the low elevation PRN 4 is now considered. The tracking performance is shown in Figure 12. MPDR beamforming applied on clean data from 10 to 15 s showed nearly $6 \mathrm{~dB}$ of gain with respect to the single antenna. In the presence of interference, MPDR was able to maintain the gain. However, the gain obtained was higher in the presence of interference and was observed to be around $12 \mathrm{~dB}$ with respect to the 
single antenna case. The $\mathrm{C} / \mathrm{N}_{0}$ gain observed with $\mathrm{PM}$ in the presence of interference was about $7 \mathrm{~dB}$. The carrier Doppler errors were computed by taking the difference between the reference antenna Doppler with clean data without interference and beamformed data in the presence of interference. The Doppler RMS errors, PLI, and C/ $\mathrm{N}_{0}$ gain for different PRNs are shown in Table 6 . These values are computed from the tracking results in the presence of interference. For all the PRNs, PLL was able to maintain lock even in the presence of interference.
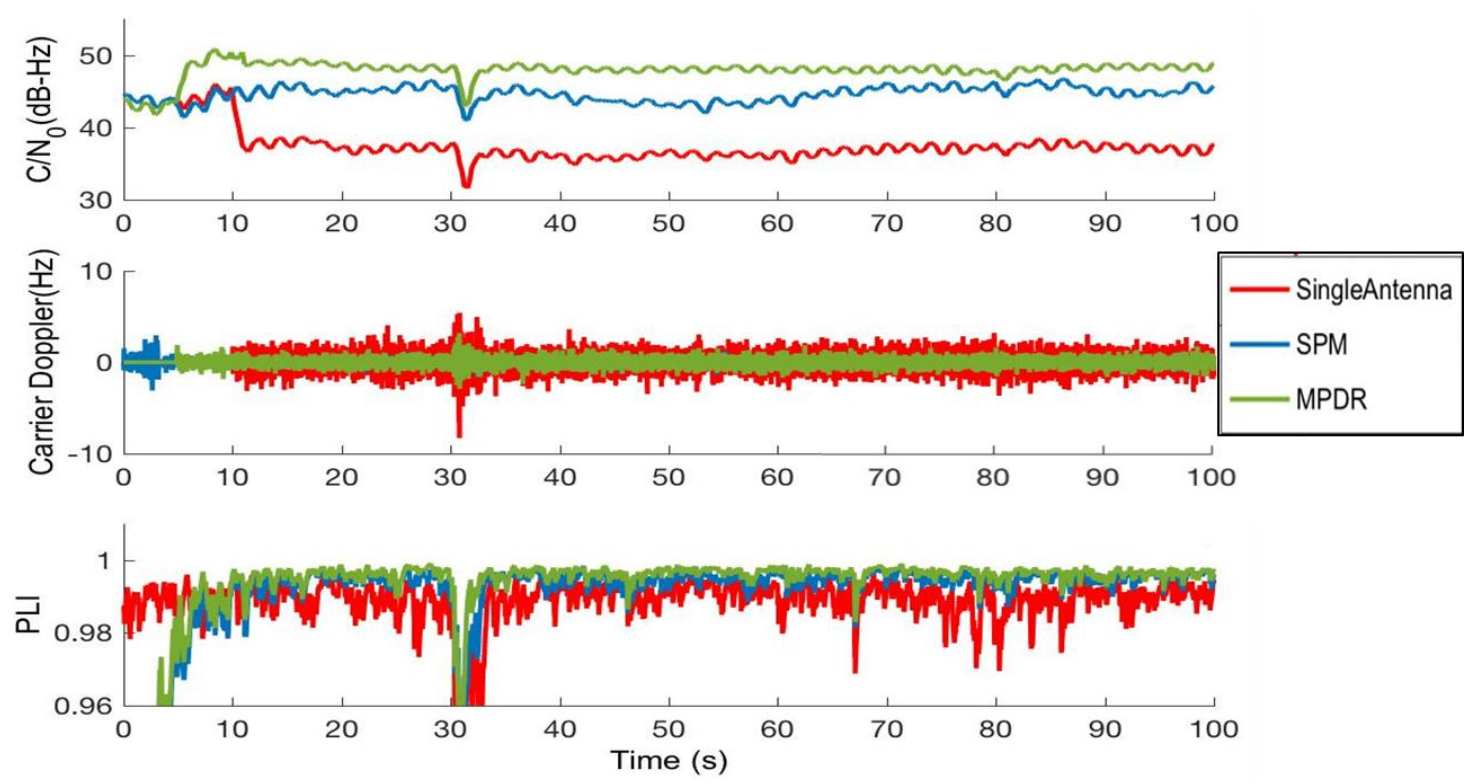

Figure 12. Tracking performance of PRN 4 with PM and MPDR using actual data with chirp interference.

Table 6. Tracking performance of different PRNs, PM and MPDR in the presence of chirp interference.

\begin{tabular}{|c|c|c|c|c|c|c|c|c|c|}
\hline \multirow[b]{2}{*}{ PRN } & \multicolumn{3}{|c|}{ Single Antenna with Interference } & \multicolumn{3}{|c|}{$\mathbf{P M}$} & \multicolumn{3}{|c|}{ MPDR } \\
\hline & $\begin{array}{l}\text { RMSE Doppler } \\
(\mathrm{Hz})\end{array}$ & PLI & $\begin{array}{c}\mathrm{C} / \mathrm{N}_{0} \\
\text { (dB-Hz) }\end{array}$ & $\begin{array}{c}\text { RMSE Doppler } \\
(\mathrm{Hz})\end{array}$ & PLI & $\begin{array}{c}\mathrm{C} / \mathrm{N}_{0} \\
(\mathrm{~dB}-\mathrm{Hz})\end{array}$ & $\begin{array}{c}\text { RMSE Doppler } \\
(\mathrm{Hz})\end{array}$ & PLI & $\begin{array}{c}\mathrm{C} / \mathrm{N}_{0} \\
(\mathrm{~dB}-\mathrm{Hz})\end{array}$ \\
\hline PRN 1 & 0.98 & 0.98 & 34.1 & 0.43 & 0.99 & 44.9 & 0.66 & 0.99 & 46.5 \\
\hline PRN 4 & 0.97 & 0.98 & 36.7 & 0.45 & 0.99 & 44.7 & 0.49 & 0.99 & 48.1 \\
\hline PRN 11 & 0.91 & 0.97 & 37.6 & 0.45 & 0.99 & 46.8 & 0.31 & 0.99 & 49.1 \\
\hline PRN 13 & 0.89 & 0.97 & 37.5 & 0.45 & 0.99 & 48.2 & 0.28 & 0.99 & 50.9 \\
\hline PRN 15 & 0.92 & 0.97 & 33.8 & 0.85 & 0.99 & 43.4 & 0.64 & 0.99 & 48.6 \\
\hline PRN 17 & 0.92 & 0.97 & 38.2 & 0.28 & 0.99 & 45.5 & 0.47 & 0.99 & 47.7 \\
\hline PRN 28 & 0.92 & 0.97 & 38.7 & 0.23 & 0.99 & 47.7 & 0.34 & 0.99 & 49.8 \\
\hline PRN 30 & 0.98 & 0.99 & 41.1 & 0.60 & 0.99 & 44.7 & 0.29 & 0.99 & 52.7 \\
\hline
\end{tabular}

\subsection{Measurement Domain Analysis}

The measurements generated by the interference free reference antenna data acts as reference measurements in this case. Differences between these measurements and those generated after beamforming were compared for PM and MPDR. The resulting time series plot of the pseudorange and carrier phase measurement errors are shown in Figure 13 and the corresponding statistics are provided in Tables 7 and 8. Pseudorange biases up to $2 \mathrm{~m}$ were observed in both PM and MPDR beamformers. The biases observed are different for different PRNs. Carrier phase biases are minimal (less than $1 \mathrm{~mm}$ ) with the MPDR beamformer for all PRNs, which agrees with the simulation results. With PM, different biases were observed for different PRNs as seen from Figure 13a and they reach $1.1 \mathrm{~cm}$ for some PRNs. The results with PM also agree with simulation results where different biases were observed for different PRNs. 

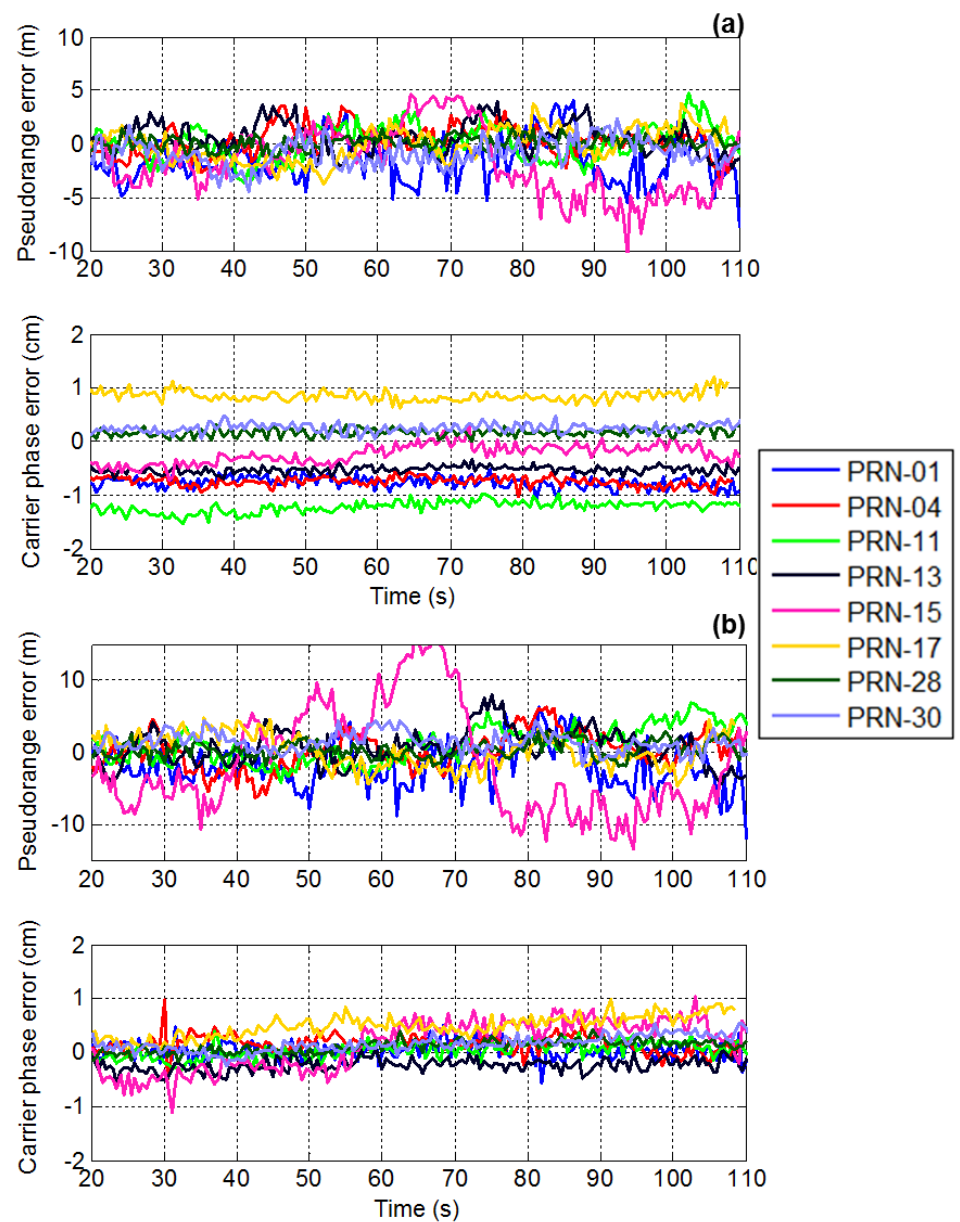

Figure 13. Pseudorange and carrier phase measurements with actual data with chirp interference for (a) PM; and (b) MPDR.

Table 7. Pseudorange measurement errors for PM and MPDR in the presence of chirp interference.

\begin{tabular}{cccccc}
\hline \multirow{2}{*}{ PRN } & \multicolumn{2}{c}{ PM } & & \multicolumn{2}{c}{ MPDR } \\
\cline { 2 - 3 } \cline { 5 - 6 } \cline { 5 - 6 } & Mean $(\mathbf{m})$ & Standard Deviation $(\mathbf{m})$ & & Mean $(\mathbf{m})$ & Standard Deviation $(\mathbf{m})$ \\
\hline PRN 1 & -1.42 & 1.94 & & -1.71 & 2.92 \\
PRN 4 & 0.34 & 1.32 & & 0.26 & 2.40 \\
PRN 11 & 0.22 & 1.49 & & 1.27 & 2.42 \\
PRN 13 & 0.48 & 1.33 & & 0.47 & 2.38 \\
PRN 15 & -1.05 & 3.31 & & -0.55 & 3.21 \\
PRN 17 & -0.06 & 1.48 & & 0.24 & 2.01 \\
PRN 28 & -0.01 & 0.69 & & 0.29 & 1.11 \\
PRN 30 & -0.69 & 1.31 & 1.12 & 1.35 \\
\hline
\end{tabular}

Table 8. Carrier phase measurement errors using PM and MPDR with chirp interference.

\begin{tabular}{cccccc}
\hline \multirow{2}{*}{ PRN } & \multicolumn{3}{c}{ PM } & & \multicolumn{2}{c}{ MPDR } \\
\cline { 2 - 3 } \cline { 5 - 6 } \cline { 5 - 6 } & Mean (mm) & Standard Deviation (mm) & & Mean (mm) & Standard Deviation (mm) \\
\hline PRN 1 & -7.3 & 2.0 & 0.3 & 1.6 \\
PRN 4 & 7.1 & 1.7 & 0.9 & 2.0 \\
PRN 11 & -10.9 & 3.8 & & 0.2 & 1.4 \\
PRN 13 & -5.0 & 1.3 & & -0.8 & 1.3 \\
PRN 15 & -2.3 & 1.8 & & 0.5 & 1.7 \\
PRN 17 & 8.2 & 1.9 & 0.9 & 2.1 \\
PRN 28 & 1.6 & 0.8 & 0.5 & 1.1 \\
PRN 30 & 2.3 & 1.1 & & \\
\hline
\end{tabular}




\subsection{Position Domain Analysis}

Carrier phase based positioning was performed using RTKLib open source software. The position errors using PM and MPDR are shown in Figure 14a and the east-north position errors scatter plot using MPDR is shown in Figure 14b. The error statistics are shown in Table 9. MPDR is able to provide fixed solutions. However, PM was not able to provide fixed solution and errors up to $1 \mathrm{~m}$ were observed.
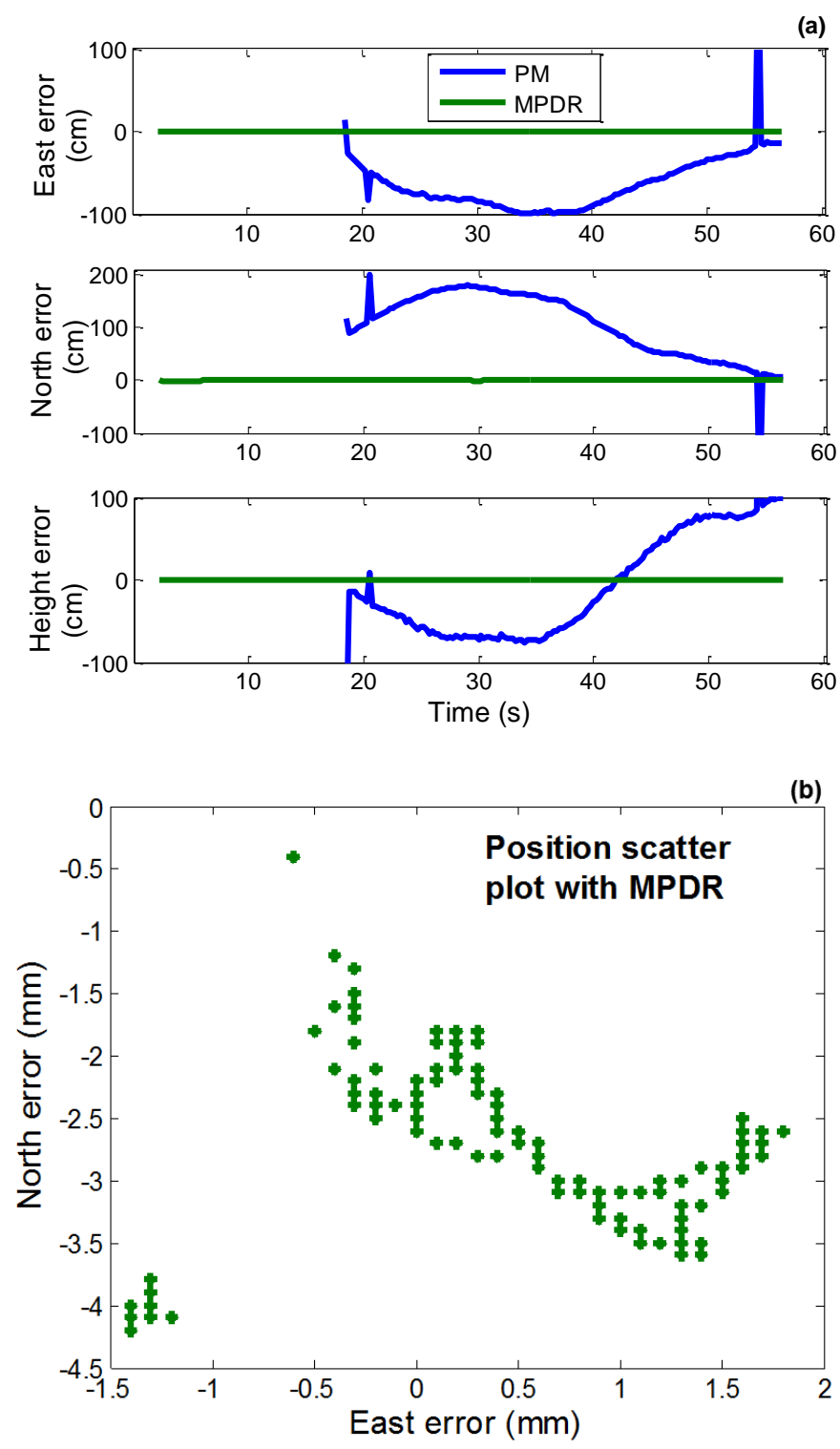

Figure 14. (a) Carrier phase based position errors with actual data with chirp interference using PM and MPDR; (b) East-north position errors scatter plot with MPDR.

Table 9. Carrier phase based position errors with actual data.

\begin{tabular}{cccccc}
\hline \multirow{2}{*}{ Position Errors } & \multicolumn{2}{c}{ PM } & & \multicolumn{2}{c}{ MPDR } \\
\cline { 2 - 3 } \cline { 5 - 6 } & Mean & Standard Deviation & & Mean & Standard Deviation \\
\hline East error $(\mathrm{cm})$ & -63.4 & 36.2 & & -0.04 & 0.08 \\
North error $(\mathrm{cm})$ & 104.5 & 65.0 & & -0.25 & 0.06 \\
Height error $(\mathrm{cm})$ & -5.09 & 78.6 & & -0.06 & 0.16 \\
\hline
\end{tabular}




\section{Conclusions}

The performance of a multi-antenna GNSS receiver under narrowband and wideband jamming conditions was investigated for high precision applications. Different types of electronic interference sources were considered for performance evaluations in terms of tracking, measurement, and position distortions for PM and MPDR beamformers. A number of GPS signal simulations were performed to evaluate measurement distortions. It was observed that MPDR did not induce measurement distortions in the simulations. With actual data, carrier phase measurement biases were minimal and pseudorange measurement errors up to $2 \mathrm{~m}$ were observed using MPDR. However, with PM, pseudorange biases up $50 \mathrm{~cm}$ were observed in the simulations and up to $2 \mathrm{~m}$ with actual data. Carrier phase measurement biases up to few $\mathrm{cm}$ were observed with PM in both simulations and actual data processing. The biases observed were different for different PRNs. Carrier phase positioning performance with MPDR was better than PM; the latter was unable to provide fixed carrier phase ambiguity solutions. With PM, position errors up to $10 \mathrm{~cm}$ were observed in simulations and up to $1 \mathrm{~m}$ with actual data.

Author Contributions: The major contribution of the paper was by Niranjana Vagle. Technical discussions and experimental tests were performed by Niranjana Vagle and Ali Broumandan. The software was developed by Niranjana Vagle and Ali Broumandan. Gérard Lachapelle supervised the work and provided technical feedback. All the authors contributed to the writing of the manuscript.

Conflicts of Interest: The authors declare no conflict of interest.

\section{Appendix A}

Tracking performance of different PRNs with CW and BWGN interference are provided in Tables A1 and A2.

Table A1. Tracking performance of different PRNs in the presence of CW interference.

\begin{tabular}{|c|c|c|c|c|c|c|c|c|c|}
\hline \multirow[b]{2}{*}{ PRN } & \multicolumn{3}{|c|}{ Single Antenna } & \multicolumn{3}{|c|}{ PM } & \multicolumn{3}{|c|}{ MPDR } \\
\hline & $\begin{array}{l}\text { RMSE Doppler } \\
(\mathrm{Hz})\end{array}$ & Mean PLI & $\begin{array}{c}\mathrm{C} / \mathrm{N}_{0} \\
(\mathrm{~dB}-\mathrm{Hz})\end{array}$ & $\begin{array}{l}\text { RMSE Doppler } \\
(\mathrm{Hz})\end{array}$ & PLI & $\begin{array}{c}\mathrm{C} / \mathrm{N}_{0} \\
(\mathrm{~dB}-\mathrm{Hz})\end{array}$ & $\begin{array}{c}\text { RMSE Doppler } \\
(\mathrm{Hz})\end{array}$ & PLI & $\begin{array}{c}\mathrm{C} / \mathrm{N}_{0} \\
(\mathrm{~dB}-\mathrm{Hz})\end{array}$ \\
\hline PRN 3 & 0.51 & 0.99 & 33.4 & 0.42 & 0.99 & 48.9 & 0.34 & 0.99 & 54.69 \\
\hline PRN 6 & 36.14 & - & 37.3 & 0.42 & 0.99 & 48.02 & 0.33 & 0.99 & 54.59 \\
\hline PRN 10 & 0.67 & 0.99 & 35.09 & 1.11 & 0.99 & 42.13 & 0.34 & 0.99 & 54.59 \\
\hline PRN 16 & 3.25 & - & 39.4 & 0.75 & 0.99 & 45.92 & 0.51 & 0.99 & 54.26 \\
\hline PRN 18 & 0.80 & 0.99 & 36.25 & 0.51 & 0.99 & 47.38 & 0.40 & 0.99 & 54.26 \\
\hline PRN 21 & 1.97 & 0.98 & 37.01 & 0.47 & 0.99 & 48.56 & 0.35 & 0.99 & 54.66 \\
\hline PRN 24 & 0.57 & 0.99 & 34.79 & 0.46 & 0.99 & 47.66 & 0.37 & 0.99 & 54.80 \\
\hline PRN 25 & 0.49 & 0.99 & 35.6 & 0.46 & 0.99 & 47.08 & 0.39 & 0.99 & 54.29 \\
\hline PRN 29 & 0.90 & 0.99 & 34.8 & 0.67 & 0.99 & 45.43 & 0.51 & 0.99 & 54.27 \\
\hline
\end{tabular}

Table A2. Tracking performances of different PRNs in the presence of BWGN interference.

\begin{tabular}{|c|c|c|c|c|c|c|c|c|c|}
\hline \multirow[b]{2}{*}{ PRN } & \multicolumn{3}{|c|}{ Single Antenna } & \multicolumn{3}{|c|}{$\mathbf{P M}$} & \multicolumn{3}{|c|}{ MPDR } \\
\hline & $\begin{array}{l}\text { RMSE Doppler } \\
(\mathrm{Hz})\end{array}$ & PLI & $\begin{array}{c}\mathrm{C} / \mathrm{N}_{0} \\
\text { (dB-Hz) }\end{array}$ & $\begin{array}{l}\text { RMSE Doppler } \\
(\mathrm{Hz})\end{array}$ & PLI & $\begin{array}{c}\mathrm{C} / \mathrm{N}_{0} \\
\text { (dB-Hz) }\end{array}$ & $\begin{array}{l}\text { RMSE Doppler } \\
(\mathrm{Hz})\end{array}$ & PLI & $\begin{array}{c}\mathrm{C} / \mathrm{N}_{0} \\
\text { (dB-Hz) }\end{array}$ \\
\hline PRN 3 & 1.10 & 0.98 & 37.7 & 0.32 & 0.99 & 48.9 & 0.26 & 0.99 & 54.4 \\
\hline PRN 6 & 1.10 & 0.98 & 37.7 & 0.36 & 0.99 & 48.0 & 0.29 & 0.99 & 54.3 \\
\hline PRN 10 & 1.03 & 0.98 & 37.7 & - & - & - & 0.35 & 0.99 & 54.0 \\
\hline PRN 16 & 1.14 & 0.98 & 37.7 & 0.46 & 0.99 & 45.9 & 0.39 & 0.99 & 54.0 \\
\hline PRN 18 & 1.08 & 0.98 & 37.7 & 0.39 & 0.99 & 47.3 & 0.29 & 0.99 & 54.0 \\
\hline PRN 21 & 1.10 & 0.98 & 37.7 & 0.35 & 0.99 & 48.5 & 0.33 & 0.99 & 54.3 \\
\hline PRN 24 & 1.15 & 0.98 & 37.7 & 0.37 & 0.99 & 47.6 & 0.28 & 0.99 & 54.5 \\
\hline PRN 25 & 1.09 & 0.98 & 37.7 & 0.40 & 0.99 & 47.0 & 0.25 & 0.99 & 54.3 \\
\hline PRN 29 & 1.17 & 0.98 & 37.7 & 0.50 & 0.99 & 45.4 & 0.46 & 0.99 & 54.0 \\
\hline
\end{tabular}

\section{Appendix B}

Measurement distortions in the presence of CW and BWGN interference are provided in this section. 
Table B1. Pseudorange measurement errors for PM and MPDR in the presence of CW interference.

\begin{tabular}{cccccc}
\hline & \multicolumn{2}{c}{ PM } & & \multicolumn{2}{c}{ MPDR } \\
\cline { 2 - 3 } \cline { 5 - 6 } PRN & Mean $(\mathbf{m})$ & Standard Deviation $(\mathbf{m})$ & & Mean $(\mathbf{m})$ & Standard Deviation $(\mathbf{m})$ \\
\hline PRN 3 & -0.11 & 0.33 & -0.06 & 0.55 \\
PRN 6 & -0.13 & 0.29 & 0.01 & 0.62 \\
PRN 10 & -0.19 & 1.73 & 0.05 & 0.80 \\
PRN 16 & -0.11 & 0.37 & -0.06 & 0.63 \\
PRN 18 & -0.14 & 0.34 & 0.03 & 0.65 \\
PRN 21 & -0.09 & 0.25 & -0.01 & 0.57 \\
PRN 24 & 0.01 & 0.49 & & 0.05 & 0.70 \\
PRN 25 & 0.07 & 0.52 & 0.03 & 0.61 \\
PRN 29 & -0.06 & 0.43 & & 0.03 & 0.69 \\
\hline
\end{tabular}

Table B2. Carrier phase measurement errors using PM and MPDR with CW interference.

\begin{tabular}{|c|c|c|c|c|}
\hline \multirow[b]{2}{*}{ PRN } & \multicolumn{2}{|r|}{ PM } & \multicolumn{2}{|r|}{ MPDR } \\
\hline & Mean (mm) & Standard Deviation (mm) & Mean (mm) & Standard Deviation (mm) \\
\hline PRN 3 & 10.9 & 0.4 & 0.01 & 0.4 \\
\hline PRN 6 & 3.4 & 0.3 & -0.01 & 0.4 \\
\hline PRN 10 & 29.6 & 1.5 & 0.07 & 0.7 \\
\hline PRN 16 & 4.8 & 0.4 & 0.05 & 0.6 \\
\hline PRN 18 & 7.5 & 0.3 & -0.01 & 0.5 \\
\hline PRN 21 & 1.5 & 0.2 & -0.05 & 0.5 \\
\hline PRN 24 & -16.0 & 0.3 & 0.01 & 0.5 \\
\hline PRN 25 & 23.1 & 0.5 & -0.03 & 0.5 \\
\hline PRN 29 & -6.7 & 0.4 & 0.03 & 0.5 \\
\hline
\end{tabular}

Table B3. Pseudorange measurement errors for PM and MPDR in the presence of BWGN interference.

\begin{tabular}{cccccc}
\hline & \multicolumn{2}{c}{ PM } & & \multicolumn{2}{c}{ MPDR } \\
\cline { 2 - 3 } \cline { 5 - 6 } PRN & Mean $(\mathbf{m})$ & Standard Deviation $(\mathbf{m})$ & & Mean $(\mathbf{m})$ & Standard Deviation $(\mathbf{m})$ \\
\hline PRN 3 & -0.11 & 0.34 & -0.06 & 0.55 \\
PRN 6 & -0.13 & 0.28 & 0.01 & 0.62 \\
PRN 10 & - & - & 0.05 & 0.81 \\
PRN 16 & -0.10 & 0.37 & -0.06 & 0.63 \\
PRN 18 & -0.14 & 0.36 & 0.03 & 0.65 \\
PRN 21 & -0.09 & 0.25 & 0.01 & 0.57 \\
PRN 24 & 0.01 & 0.49 & 0.05 & 0.70 \\
PRN 25 & 0.07 & 0.53 & -0.04 & 0.61 \\
PRN 29 & -0.06 & 0.45 & & 0.04 & 0.69 \\
\hline
\end{tabular}

Table B4. Carrier phase measurement errors using PM and MPDR with BWGN interference.

\begin{tabular}{cccccc}
\hline & \multicolumn{2}{c}{ PM } & & \multicolumn{2}{c}{ MPDR } \\
\cline { 2 - 3 } \cline { 5 - 6 } PRN & Mean $(\mathbf{m m})$ & Standard Deviation $\mathbf{( m m})$ & & Mean $(\mathbf{m m})$ & Standard Deviation (mm) \\
\hline PRN 3 & 10.9 & 0.4 & 0.01 & 0.5 \\
PRN 6 & 3.4 & 0.3 & -0.01 & 0.5 \\
PRN 10 & - & - & 0.07 & 0.7 \\
PRN 16 & 4.8 & 0.4 & 0.05 & 0.6 \\
PRN 18 & 7.5 & 0.3 & -0.01 & 0.5 \\
PRN 21 & 1.5 & 0.2 & -0.05 & 0.5 \\
PRN 24 & -16.0 & 0.4 & 0.01 & 0.5 \\
PRN 25 & 23.1 & 0.5 & -0.03 & 0.5 \\
PRN 29 & -6.7 & 0.4 & & 0.03 & 0.5 \\
\hline
\end{tabular}




\section{References}

1. Fernández-Prades, C.; Arribas, J.; Closas, P. Robust GNSS Receivers by Array Signal Processing: Theory and Implementation. Proc. IEEE 2016, 104, 1207-1220. [CrossRef]

2. Gupta, I.J.; Weiss, I.M.; Morrison, A.W. Desired Features of Adaptive Antenna Arrays for GNSS Receivers. Proc. IEEE 2016, 104, 1195-1206. [CrossRef]

3. Broumandan, A.; Jafarnia-Jahromi, A.; Daneshmand, S.; Lachapelle, G. Overview of Spatial Processing Approaches for GNSS Structural Interference Detection and Mitigation. Proc. IEEE 2016, 104, 1246-1257. [CrossRef]

4. Cuntz, M.; Konovaltsev, A.; Meurer, M. Concepts, Development, and Validation of Multiantenna GNSS Receivers for Resilient Navigation. Proc. IEEE 2016, 104, 1288-1301. [CrossRef]

5. Amin, M.G.; Wang, X.; Zhang, Y.D.; Ahmad, F.; Aboutanios, E. Sparse Arrays and Sampling for Interference Mitigation and DOA Estimation in GNSS. Proc. IEEE 2016, 104, 1302-1317. [CrossRef]

6. Daneshmand, S.; Jafarnia-Jahromi, A.; Broumandan, A.; Lachapelle, G. A GNSS structural interference mitigation technique using antenna array processing. In Proceedings of the 2014 IEEE 8th Sensor Array and Multichannel Signal Processing Workshop (SAM), A Coruna, Spain, 22-25 June 2014; pp. 109-112.

7. Arribas, J.; Closas, P.; Fernández-Prades, C. Interference mitigation in GNSS receivers by array signal processing: A software radio approach. In Proceedings of the 2014 IEEE 8th Sensor Array and Multichannel Signal Processing Workshop (SAM), A Coruna, Spain, 22-25 June 2014; pp. 121-124.

8. Kalyanaraman, S.K.; Braasch, M.S. GPS Adaptive Array Phase Compensation Using a Software Radio Architecture. Navigation 2010, 57, 53-68. [CrossRef]

9. Sahmoudi, M.; Amin, M. Optimal robust beamforming for interference and multipath mitigation in GNSS arrays. In Proceedings of the IEEE International Conference on Acoustics, Speech and Signal Processing, Honolulu, HI, USA, 15-20 April 2007; Volume 3, pp. 693-696.

10. Seco-Granados, G.; Fernández-Rubio, J.; Fernández-Prades, C. ML estimator and hybrid beamformer for multipath and interference mitigation in GNSS receivers. IEEE Trans. Signal Process. 2005, 53, 1194-1208. [CrossRef]

11. Amin, M.G.; Sun, W. A novel interference suppression scheme for global navigation satellite systems using antenna array. IEEE J. Sel. Areas Commun. 2005, 23, 999-1012. [CrossRef]

12. Chuang, Y.C. (Jay); Buchanan, M.; O’Brien, A.; Gupta, I.J. Prediction of Antenna Induced Biases for GNSS Receivers. In Proceedings of the International Technical Meeting of the Institute of Navigation, San Diego, CA, USA, 27-29 January 2014.

13. Chuang, Y.C. (Jay); Gupta, I.J. Antenna Induced Biases in GNSS Receiver Measurements. In Proceedings of the International Technical Meeting of the Institute of Navigation, San Diego, CA, USA, 28-30 January 2013; pp. 164-171.

14. McGraw, G.A.; McDowell, C.; Kelly, J.M. GPS Anti-Jam Antenna System Measurement Error Characterization and Compensation. In Proceedings of the 19th International Technical Meeting of the Satellite Division of the Institute of Navigation (ION GNSS 2006), Fort Worth, TX, USA, 26-29 September 2006; pp. 705-714.

15. McGraw, G.A.; McDowell, C.; Young, S.Y.; Glessner, G.W. Assessment of GPS Anti-Jam System Pseudorange and Carrier Phase Measurement Error Effects. In Proceedings of the 18th International Technical Meeting of the Satellite Division of the Institute of Navigation (ION GNSS 2005), Long Beach, CA, USA, 13-16 September 2005; pp. 603-617.

16. McGraw, G.A.; Young, S.Y.; Reichenauer, K. Evaluation of GPS Anti-Jam System Effects on Pseudorange and Carrier Phase Measurements for Precision Approach and Landing. In Proceedings of the 17th International Technical Meeting of the Satellite Division of the Institute of Navigation (ION GNSS 2004), Long Beach, CA, USA, 21-24 September 2004; pp. 2742-2751.

17. Vagle, N.; Broumandan, A.; Jafarnia-Jahromi, A.; Lachapelle, G. Characterization of GNSS Measurement Distortions Due to Antenna Array Processing in the Presence of Interference Signals. In Proceedings of Ubiquitous Positioning Indoor Navigation and Location Based Service, Corpus Christi, TX, USA, 20-21 November 2014.

18. Kim, U.S.; De Lorenzo, D.S.; Akos, D.; Gautier, J.; Enge, P.; Orr, J. Precise phase calibration of a controlled reception pattern GPS antenna for JPALS. In Proceedings of the Position Location and Navigation Symposium, Monterey, CA, USA, 26-29 April 2004; pp. 478-485. 
19. Kim, U.S. Mitigation of Signal Biases Introduced by Controlled Reception Pattern Antennas in a High Integrity Carrier Phase Differential GPS System. Ph.D. Thesis, Stanford University, Stanford, CA, USA, March 2007.

20. Church, C.M.; Gupta, I.J. Estimation of Adaptive Antenna Induced Code and Carrier Phase Bias in GNSS Receivers. Navigation 2009, 56, 151-160. [CrossRef]

21. Griffith, K.A.; Gupta, I.J. Effect of mutual coupling on the performance of GPS AJ antennas. In Proceedings of the IEEE/ION Position, Location and Navigation Symposium, Monterey, CA, USA, 5-8 May 2008; pp. 871-877.

22. Kalyanaraman, S.K.; Braasch, M.S. Tight Integration of a GPS Adaptive Array with a Software-Defined Receiver. In Proceedings of the 2007 National Technical Meeting of The Institute of Navigation, San Diego, CA, USA, 22-24 January 2007; pp. 657-668.

23. Zhang, Y.; Amin, M. Anti-jamming GPS receiver with reduced phase distortions. IEEE Signal Process. Lett. 2012, 19, 635-638. [CrossRef]

24. Van Trees, H.L. Optimum Array Processing, Detection, Estimation, and Modulation Theory Part IV; John Wiley \& Sons: New York, NY, USA, 2002; pp. 428-699.

25. Vagle, N.; Broumandan, A.; Jafarnia-Jahromi, A.; Lachapelle, G. Performance of Antenna Array Calibration in Multipath Environments. In Proceedings of the ION International Technical Meeting, Monterey, CA, USA, 25-28 January 2016.

26. Petovello, M.G.; O'Driscoll, C.; Lachapelle, G.; Borio, D.; Murtaza, H. Architecture and Benefits of an Advanced GNSS Software Receiver. J. Glob. Position. Syst. 2008, 7, 156-168. [CrossRef]

27. Zoltowski, M.D.; Gecan, A.S. Advanced adaptive null steering concepts for GPS. In Proceedings of the Military Communications Conference, San Diego, CA, USA, 5-8 November 1995; pp. 1214-1218.

28. Spirent Communications PLC. Simgen Software User Manual; Spirent Communications PLC: Paignton, UK, 2012.

29. Van Dierendonck, A.J.; Parkinson, B.W.; Spilker, J.J., Jr. Global Positioning System: Theory and Applications; American Institute of Aeronautics: Washington, DC, USA, 1996; Volume 1, pp. 393-394.

30. Takasu, T. RTKLIB: An Open Source Program Package for GNSS Positioning. Available online: http://www.rtklib.com/ (accessed on 23 September 2016).

31. Novatel GPSAntennaTM Model 501. Available online: http://www.novatel.com/assets/Documents/ Manuals/om-20000001.pdf (accessed on 10 April 2016).

(C) 2016 by the authors; licensee MDPI, Basel, Switzerland. This article is an open access article distributed under the terms and conditions of the Creative Commons Attribution (CC-BY) license (http:/ / creativecommons.org/licenses/by/4.0/). 